Authors: Adam Pietrobon ${ }^{1,2,3}$, Julien Yockell-Lelièvre ${ }^{1,3}$, Nicole Melong ${ }^{4}$, Laura J. Smith ${ }^{5,6,7}$, Sean P. Delaney ${ }^{1,2,3}$, Nadine Azzam ${ }^{4}$, Chang Xue ${ }^{6,7}$, Nishanth Merwin ${ }^{8}$, Eric Lian ${ }^{1,2,3}$, Alberto Camacho-Magallanes ${ }^{1,2,3}$, Carole Doré ${ }^{1}$, Gabriel Musso ${ }^{8}$, Lisa M. Julian ${ }^{9}$, Arnold S. Kristof ${ }^{10}$, Roger Y. Tam ${ }^{11}$, Jason N. Berman ${ }^{2,4}$, Molly S. Shoichet ${ }^{5,6,7,12}$, William L. Stanford ${ }^{1,2,3 *}$

\title{
Three-dimensional drug screen identifies HDAC inhibitors as therapeutic agents in mTORC1-driven lymphangioleiomyomatosis
}

\section{Affiliations:}

$10{ }^{1}$ The Sprott Centre for Stem Cell Research, Regenerative Medicine Program, Ottawa Hospital

11 Research Institute; Ottawa, Canada.

$12{ }^{2}$ Department of Cellular and Molecular Medicine, University of Ottawa; Ottawa, Canada.

$13{ }^{3}$ Ottawa Institute of Systems Biology; Ottawa, Canada.

$14{ }^{4}$ Department of Pediatrics, CHEO Research Institute; Ottawa, Canada.

$15{ }^{5}$ Department of Chemical Engineering and Applied Chemistry, University of Toronto; Toronto,

16 Canada.

$17{ }^{6}$ Institute for Biomaterials and Biomedical Engineering, University of Toronto; Toronto,

18 Canada.

${ }^{7}$ The Donnelly Centre for Cellular and Biomolecular Research; Toronto, Canada.

${ }^{8}$ BioSymetrics, Inc; Toronto, Canada.

${ }^{9}$ Centre for Cell Biology, Development, and Disease, Department of Biological Sciences, Simon Fraser University; Burnaby, Canada.

${ }^{10}$ Meakins-Christie Laboratories and Translational Research in Respiratory Diseases Program, Research Institute of the McGill University Health Centre, Faculty of Medicine, Departments of Medicine and Critical Care; Montreal, Canada.

${ }^{11}$ Centre for Biologics Evaluation, Biologic and Radiopharmaceutical Drugs Directorate, Health Canada; Ottawa, Canada.

${ }^{12}$ Department of Chemistry, University of Toronto; Toronto, Canada.

* Corresponding author

31 Dr. William L. Stanford, Ph.D.

32 The Ottawa Hospital, 501 Smyth Rd, Box 511, CCW 5206c

33 Ottawa, ON K1H 8L6, Canada

34 wstanford@ohri.ca

One Sentence Summary: We performed a drug screen in 3D and discovered HDAC inhibitors exhibit therapeutic efficacy in models of the lung disease lymphangioleiomyomatosis. 


\section{ABSTRACT}

Lymphangioleiomyomatosis (LAM) is a rare disease involving cystic lung destruction by

40 invasive LAM cells. These cells harbor loss-of-function mutations in TSC2, conferring

41 constitutive mTORC1 signaling. Rapamycin is the only clinically approved disease-modifying

42 treatment, but its action is cytostatic and disease progresses upon its withdrawal. There is a

43 critical need to identify novel agents that prevent the invasive phenotype and/or eradicate the

44 neoplastic LAM cells. Here, we employed novel cellular and extracellular models to screen for

45 candidate therapeutics in a physiologically relevant setting. We observed that lung-mimetic

46 hydrogel culture of pluripotent stem cell-derived diseased cells more faithfully recapitulates

47 human LAM biology compared to conventional culture on two-dimensional tissue culture

48 plastic. Leveraging our culture system, we conducted a three-dimensional drug screen using a

49 custom 800-compound library, tracking cytotoxicity and invasion modulation phenotypes at the

50 single cell level. We identified histone deacetylase (HDAC) inhibitors as a group of anti-invasive

51 agents that are also selectively cytotoxic towards $T S C 2^{-/-}$cells. Unexpectedly, we observed that

52 next generation ATP-competitive mTORC1/2 inhibitors potentiate invasion. We determined anti-

53 invasive effects of HDAC inhibitors to be independent of genotype, while selective cell death is

54 mTORC1-dependent and mediated by apoptosis. Drug performance was subsequently evaluated

55 at the single cell level in zebrafish xenografts. We observed consistent therapeutic efficacy in

56 vivo at equivalent concentrations to those used in vitro, substantiating HDAC inhibitors as

57 potential therapeutic candidates for pursuit in patients with LAM. 


\section{INTRODUCTION}

Lymphangioleiomyomatosis (LAM) is a cystic lung disease predominately affecting women, at a prevalence of 1 to 10 per million (1). LAM can occur sporadically or in association with the multisystem tumor-forming disorder, Tuberous Sclerosis Complex (TSC) (2). The

62 pulmonary histopathology is characterized by microscopic nodules consisting of immature

63 smooth muscle-like cells that express markers of neural crest lineages (3). These invading cells

64 digest the lung parenchyma forming cystic lesions that lead to progressive respiratory decline

65 and fatality if untreated (4-6). The molecular etiology of LAM involves loss-of-function

66 mutations in the endogenous mTORC1 suppressor TSC2, thereby inducing hyperactivation of

67 mTORC1 anabolic and tumorigenic signalling (7). The allosteric mTORC1-inhibitor rapamycin

68 (clinically, sirolimus) slows disease progression and improves symptomatology (8-11). While

69 clinical approval of rapamycin by the FDA in 2015 has led to a dramatic new frontier in the

70 LAM therapeutic landscape, significant limitations exist. A subset of patients do not respond to

71 treatment, and rapamycin is invariably cytostatic, with rapid disease progression upon treatment

72 withdrawal $(11,12)$. There is a critical need to discover novel treatment strategies that can

73 eradicate LAM cells.

A key step in the pathway to therapeutic development is the effective modelling of

75 disease characteristics. In this domain, LAM has remained a challenge. Cultures of cells derived

76 from human pulmonary LAM lesions grow as a heterogeneous mixture with rapid exhaustion of

$77 \mathrm{TSC}^{-/}$cells, prohibiting the establishment of clonal primary cell lines (13). While a genome

78 engineering strategy would seem straightforward for this monogenic disease, the cell-of-origin of

79 LAM remains unknown, begging the question of which cell type to engineer. While we have

80 demonstrated that $\mathrm{TSC}^{-/}$human pluripotent stem cell-derived neural crest cells model several 
81 phenotypic features of LAM (14), neural crest cells consist of a diverse and plastic population

82 that are not readily scalable for drug screening purposes. Animal models of LAM have been

83 comparably challenging to establish, and none to date have recapitulated pathognomonic features

84 such as histological premelanosome protein (PMEL) positivity and concomitant elevated serum

85 levels of vascular endothelial growth factor D (VEGF-D) (15).

An emerging consideration in disease modelling is the contribution of the extracellular matrix (ECM) to disease biology. Water-swollen networks of polymers termed hydrogels have arisen as effective tools for mimicking salient elements of the native ECM while exhibiting mechanics similar to many soft tissues (16). Hydrogels can be broadly classified as either natural, synthetic, or hybrid materials. One such hybrid scaffold is hyaluronic acid, a naturally-

91 sourced material that can be readily modified to independently tune ECM features of interest,

92 such as elasticity, stiffness, and viscosity (17). A viscoelastic hydrogel with a derivatized

93 hyaluronic acid backbone has been shown to permit the study of invasive properties of LAM

94 cellular models in three-dimensional culture (18). Importantly, three-dimensional culture systems

95 have been demonstrated as more predictive of in vivo drug responses compared to conventional

96 culture on two-dimensional plastic $(19,20)$.

97 In recent years, there has been a resurgence of interest in phenotype-based screens for

98 drug discovery compared to target-based approaches (21). An analysis of therapeutics approved

99 between 1999 and 2008 revealed that $62 \%$ first-in-class drugs were discovered by phenotype-

100 based screens, despite the fact that such screens represented only a small subset of the overall

101 total (22). The apparent superiority of phenotype-based approaches may in part arise from the

102 ability to identify compounds which exhibit a therapeutic effect by modulating multiple targets

103 simultaneously (21). In addition, phenotypic drug screens can be multiplexed with counter- 
104 screening, ensuring candidate therapeutics do not also confer undesirable side-effects, such as

105 physiological toxicity. In the context of LAM, a monogenetic disease, this counter-screening

106 takes shape by directly comparing $\operatorname{TSC}^{-/-}$cells against matched wild type (WT) controls.

107 Here, we analyze a novel hydrogel culture system of pluripotent stem cell-derived

108 models, and observe the cell type employed, genotype, and culture substrate all contribute to

109 modelling features of LAM. We performed a three-dimensional drug screen, tracking

110 cytotoxicity and invasion modulation phenotypes at the single cell level. We identified histone

111 deacetylase (HDAC) inhibitors as anti-invasive and selectively cytotoxic towards TSC $^{\%}$ cells.

112 Importantly, we observed consistent therapeutic efficacy upon xenotransplantation of human cell

113 models into zebrafish larvae, highlighting HDAC inhibitors as potential therapeutic candidates

114 for pursuit in patients. 


\section{RESULTS}

\section{Stem cell-derived models exhibit features of LAM, independent of genotype}

118 established primary cell lines by in vivo differentiation of human pluripotent stem cells (hPSCs),

119 as previously described (23). Briefly, hPSCs were injected into NOD.Cg-Prkdc ${ }^{s c i d} I l 2 r g^{t m l W j l} / \mathrm{SzJ}$

120 (NSG) immunodeficient mice to form teratomas, which were explanted and expanded in smooth

121 muscle-cell enriching conditions (Fig. S1A). We used a previously reported isogenic pair of

122 female mCherry ${ }^{+}$WT and genome-engineered $T S C 2^{-/-}$hPSCs (14). Cell cultures exhibit a

123 predominately spindle cell morphology and express $\alpha$-smooth muscle actin (ACTA2) protein in

124 all isolated cells (Fig. 1A, Fig. S1B). Further, immunofluorescence analysis identified a small

125 fraction of $\mathrm{PMEL}^{+}$cells $(\sim 0.13 \%)$, a hallmark marker of pulmonary LAM (Fig. 1A, Fig. S1C).

126 The high fraction of ACTA2 ${ }^{+}$and low fraction of $\mathrm{PMEL}^{+}$cells in culture is consistent with the

127 relative abundance of these markers in heterogenous human LAM lesions (3). Notably, the

128 percentage of $\mathrm{PMEL}^{+}$and $\mathrm{ACTA}^{+}$cells did not vary between WT and TSC2 ${ }^{-/}$(Fig. S1B-C).

129 Secreted VEGF-D, a critical biochemical biomarker used in the diagnosis of LAM, was detected

130 in the supernatant of both WT and $T S C 2^{--}$cultures and was insensitive to acute rapamycin

131 treatment (Fig. 1B). Together, these data suggest the cell models employed exhibit features of

132 LAM as a product of the cell type isolated, independent of genotype.

134 Three-dimensional hydrogel culture enables study of the LAM invasive phenotype at single

135 cell resolution

136 We next sought to model the pulmonary invasive phenotype of LAM cells by adapting a

137 lung-mimetic hydrogel culture system (18). The hydrogel is synthesized by crosslinking 
138 hyaluronic acid strands with matrix metalloprotease (MMP)-cleavable peptides, while

139 embedding vitronectin peptides and methylcellulose to increase cell adhesion and matrix

140 plasticity, respectively. Cells are plated on top of the synthesized hydrogel and actively invade

141 through the material (Fig. 1C-D, Supplementary Movies 1-2). By staining with a nuclear dye and

142 acquiring multiplanar images through the optically clear hydrogel, we identify every cell in XYZ

143 planes and compute invasion distances at single cell resolution (Fig. S1D).

144 We observed all cells from both WT and $T S C 2^{-/-}$cultures to invade through the hydrogel,

145 albeit at variable distances (Fig. 1E). On average, WT cultures invaded further than $T S C 2^{-/}$in a

146 manner insensitive to acute rapamycin treatment (Fig. 1F). We posited that differing invasion

147 distances of cells in the same culture reflect a cell autonomous property, rather than a reflection

148 of stochasticity. To test this, we isolated and expanded clones from WT and $T S C 2^{-/}$bulk cultures

149 and subjected these clones to hydrogel culture. We observed a subset of clones with dramatically

150 high invasion speeds, and likewise, a subset with slow invasion speeds (Fig. S1E). These data

151 suggest differential cell autonomous capacities for invasion in putative heterogenous cultures.

152 Finally, we investigated modes of invasion employed by LAM cell models in this hydrogel

153 system. Similar to previous findings (18), we observed a decrease in invasion upon treatment

154 with the pan-MMP inhibitor GM6001 or the Rho-kinase (ROCK) inhibitor Y27632, indicating

155 both protease-dependent and independent modes of invasion employed (Fig. 1G).

\section{Loss of TSC2 and hydrogel culture both confer transcriptomic features of LAM}

To profile our cell culture system more comprehensively, we conducted bulk RNA-seq of

$159 \mathrm{WT}$ and $\mathrm{TSC}^{-/-}$cells, in the presence or absence of rapamycin, and in both plastic and hydrogel

160 culture, for a total of 8 sample conditions (Fig. 1H). Principal components analysis (PCA) 
161 revealed sample genotype to be driving the primary axis of variation, and culture substrate to be

162 driving the secondary axis of variation (Fig. 1I). Rapamycin treatment induced a substantial

163 global transcriptomic change in the $T S C 2^{-/}$cells, inducing a profile more similar to WT cells

164 (Fig. 1I).

We conducted differential expression analysis comparing across genotype ( $\operatorname{TSC}^{-/-}$vs.

166 WT) and culture substrate (hydrogel vs. plastic) in untreated samples, while holding the

167 reciprocal covariate constant. At a false discovery rate (FDR) $<0.05$, we identified 6,317

168 differentially expressed genes (DEGs, 1,793 with $\left|\log _{2} \mathrm{FC}\right|>1$ ) between WT and TSC2 $^{-/}$, and

4,432 DEGs (771 with $\left|\log _{2} \mathrm{FC}\right|>1$ ) between plastic and hydrogel (Table S1A-B, Fig. S1F-G).

170 While exhibiting some overlap, these DEG lists were largely distinct (Fig. S1H). We found

$17178.8 \%$ of the DEGs distinguishing genotype to be reversed by rapamycin treatment, suggesting

172 mTORC1-dependency (Fig. 1J, left panel). In contrast, the expression of DEGs distinguishing

173 plastic versus hydrogel cultures remained largely unchanged in the presence of rapamycin (Fig.

$1741 \mathrm{~J}$, right panel). We next examined the overlap of these DEGs with a recently published LAM

175 gene signature derived from single cell RNA-seq profiling of primary lesions (24). We observed

176 that both DEG lists overlap substantially (65.8\% of the total 760 LAM genes) and share both

177 common and distinct genes with the LAM gene signature (Fig. 1K).

178 To glean further biological insight, we conducted GO term enrichment (Table S2A-B,

179 Fig. S1I-J). Both DEG lists ranked “extracellular matrix organization” as most highly enriched,

180 which is also the top enriched term in a primary LAM lesion gene signature list (24). The DEGs

181 distinguishing genotype were also enriched in many terms related to development, similar to

182 primary LAM lesions (24). Interestingly, the DEGs distinguishing culture substrates were largely

183 enriched in terms related to proliferation (Table S1B, Fig. S1J). LAM is an indolent disease 
184 which progresses at a slow pace relative to other invasive diseases; only a small fraction of cells

185 actively proliferative in primary LAM lesions (3). On plastic, we found LAM cell models

186 proliferated rapidly, with $\sim 30 \%$ of cells incorporating EdU after a short 3-hour pulse (Fig. 1L).

187 In contrast, $T S C 2^{\%-}$ cells proliferated at a slightly slower pace, consistent with previous studies of

188 loss of TSC2 in primary cells (25). Acute rapamycin treatment reduced proliferation of $T S C 2^{-/-}$

189 cells but did not have a detectable effect on WT cultures. However, subjecting cells to hydrogel

190 culture caused a dramatic decrease in cell proliferation (Fig. 1L), likely reflective of the

191 proliferation-invasion dichotomy (26). Together, these data suggest both genotype (loss of TSC2)

192 and culture substrate (3D hydrogel) induce transcriptomic landscapes which model LAM

193 features.

Hydrogel culture potentiates differential mTORC1-signalling between WT and TSC2 ${ }^{-/-}$cells

mTORC1 hyperactivation is a hallmark feature of primary LAM lesions compared to

normal adjacent WT tissue. To assess mTORC1 signalling status, we performed a low input

201 (Fig. 2A). Remarkably, culturing on hydrogel potentiated a dramatic difference in mTORC1

202 signalling, with WT cells downregulating activity to rapamycin-treated levels and $T S C 2^{-/-}$cells

203 upregulating signalling above levels seen on plastic alone. This is consistent with the PCA of

204 transcriptomic landscapes, whereby WT untreated and WT rapamycin-treated samples from

205 hydrogel culture cluster slightly more closely compared to plastic culture (Fig. 1I). To

206 corroborate these findings at the single cell level, we examined mTORC1 signalling by 
immunofluorescence (Fig. 2B, S2A). While a small difference in mTORC1 signalling was observed between WT and $T S C 2^{-/-}$cells cultured on plastic, this difference was potentiated in 3D hydrogel culture. Importantly, mTORC1 signalling in WT cells was seen to mirror rapamycintreated levels only when cultured on hydrogel.

212 by interrogation of our bulk RNA-seq dataset. To do so, we tested for genes with a significant

213 coefficient fit to the genotype:substrate interaction term (See Supplementary Materials and

214 Methods) and identified 761 DEGs at FDR < 0.05 (Table S1C). Network analysis of GO terms

215 enriched in this DEG list revealed two principal nodes, one related to sterol synthesis and the

216 other to ribonucleotide metabolism (Fig. 2C, Table S2C). Notably, both these metabolic

217 pathways have been associated with mTORC1 activity (27).

219 differ between plastic and hydrogel culture, we clustered the 761 DEGs based on their

220 expression pattern across the 8 experimental conditions (Fig. S2B). Strikingly, genes related to

221 sterol synthesis and ribonucleotide metabolism partitioned largely into two distinct clusters (Fig.

222 S2B). We next classified each gene cluster into one of three categories based on the magnitude

223 of expression differences between WT and $T S C 2^{\%}$, and whether the expression changes were

224 rescued by rapamycin (Fig. S2C). Remarkably, we find that $69 \%$ of the 761 DEGs showed a

225 greater (or a unique) difference between WT and $T S C 2^{-/-}$cells in hydrogel culture compared to

226 plastic, which was rescued by rapamycin (Fig. 2D). Together, these results demonstrate that

227 hydrogel culture potentiates differential mTORC1 signalling between WT and TSC2 $2^{-/}$cells,

228 reinforcing a physiologically relevant environment in which mTORC1-dependent phenotypes

229 can be identified. 
231 Three-dimensional drug screen identifies compounds that modulate invasion and cell

232 viability

233 We next employed our hydrogel culture system to identify potential therapeutic

234 compounds. Cell death was measured at the single cell level by application of the live cell

235 imaging fluorophore SyTOX, which selectively permeates cells with compromised plasma

236 membrane integrity. We first tested a known cytotoxic compound, the proteasome inhibitor

237 carfilzomib, and identified substantial cell death by live cell imaging (Fig. 3A, S3A).

238 Additionally, we confirmed the ability to detect invasion modulation effects at the single cell

239 level by employing the known anti-invasion Src kinase inhibitor dasatinib (Fig. 3B, S3B). To

240 achieve the throughput necessary for a therapeutic screen, we acquired live cell images by high

241 content microscopy paired with automated image analysis tools developed in house. We

242 calculated the drug screen Z' (a metric for assay quality) to be 0.873 for cytotoxicity

243 measurements and 0.533 for invasion modulation. We subsequently screened a curated library of

244800 structurally diverse, bioactive, membrane-penetrant compounds (Fig. 3C, Table S3A). Of

245 these compounds, 39\% have been trialed and shown to be safe for use in humans. We tested each

246 compound on both WT and $\mathrm{TSC}^{-/}$cells in the presence and absence of rapamycin to elucidate

247 mTORC1-dependency.

$248 \quad$ We found a wide variety of compounds with invasion modulatory and cytotoxic

249 capabilities (Table S3B-C). Unsurprisingly, highly cytotoxic compounds also led to a reduction

250 in bulk invasion (Fig. 3D). This trend was independent of genotype and rapamycin treatment

251 (Fig. S3C). However, we observed many compounds which conferred an anti-invasive effect in

252 the absence of detectable cytotoxicity (Fig. 3D, S3C). We next computed therapeutic invasion z- 
scores (i.e., statistical measure of compound effect size) by comparing against the vehicle control

254 invasion distribution. Remarkably, while we identified several anti-invasive compounds,

255 numerous compounds significantly increased invasion (Fig. 3E-F), a phenotype that would be

256 otherwise overlooked if screening on two-dimensional plastic and could lead to severe adverse

257 consequences in the clinical setting. In general, more compounds in this library were identified to

258 significantly attenuate rather than potentiate invasion (Fig. 3F). Importantly, we observed a

259 substantial overlap in the compounds identified to be anti-invasive across genotypes and

260 treatment conditions, with very few drugs demonstrating a genotype-selective block to invasion

261 (Fig. 3G, S3D). Together, these data demonstrate the identification of a collection of compounds

262 which block invasion in these cell populations, irrespective of TSC2 genotype.

263 A key goal in the therapeutic development landscape for LAM is the identification of

264 compounds which exert selective cytotoxicity towards $T S C 2^{--}$cells. Interestingly, we observed

265 that $T S C 2^{-/}$cells exhibited pan-compound resistance, with over 7-fold more compounds

266 demonstrating significant cytotoxicity towards WT compared to $T S C 2^{-/}$cells (Fig. 3H). This

267 selectivity is reduced to half with the addition of rapamycin, suggesting generalized resistance is

268 largely due to mTORC1 hyperactivation in $T S C 2^{-/-}$cells. (Fig. 3H). We compared the list of

269 compounds that are selectively cytotoxic towards $T S C 2^{-/}$cells in the presence versus absence of

270 rapamycin, and observed only a $15 \%$ overlap, indicating therapeutic vulnerabilities vary

271 depending on mTORC1 signalling activity (Fig. 3I). In summary, we identified a suite of anti-

272 invasive and selectively cytotoxic therapeutics which can be mined for further development in

273 LAM (Table S3B-C).

274 


\section{Enrichment analysis predicts HDAC inhibitors as anti-invasive and selectively cytotoxic}

\section{6 towards TSC2 $^{-/-}$cells}

To refine our small molecule list for further investigation, we sought to identify

278 outperforming compounds which modulate targets of the same class. Using the known annotated

279 targets of the employed compounds, we performed target enrichment analysis by adapting the

280 Gene Set Enrichment Analysis (GSEA) algorithm. We identified targets conferring well-

281 established selective cytotoxicity towards $\operatorname{TSC}^{-/}$and anti-invasive classes, including

282 proteasome inhibition (cytotoxicity) and Src and Rho kinase inhibition (anti-invasive) (Fig. S3E-

283 F, Table S4A-D). Of note, Src inhibition, a therapeutic route explored in LAM, was found to be

284 selectively cytotoxic towards WT cells (Fig. S3E). Remarkably, pan-HDAC inhibition was

285 observed to be the only class in the top 10 most significant annotations for selective cytotoxicity

286 towards $\mathrm{TSC}^{-/}$and generalized anti-invasion. We note a substantial favourable enrichment of

287 HDAC-targeting compounds by both metrics, however, not all compounds annotated to inhibit

288 HDACs performed favourably (Fig 3J).

A limiting factor to our analyses was the small number of compounds which were

290 identified to selectively eliminate $T S C 2^{-/-}$cells. We sought to extend our compound list in silico

291 using a structure-based approach with a mechanism of action prediction algorithm (Elion $\left.{ }^{\mathrm{TM}}\right)$. In

292 brief, chemical features are extracted from compound structures and matched with screen

293 performance values to train a machine learning algorithm for prediction of other possibly

294 efficacious compounds. Compounds predicted to be efficacious in silico are then analyzed by

295 target enrichment and pathway analysis. Using this approach, we corroborated HDACs as highly

296 enriched targets for both selective cytotoxicity and anti-invasion (Table S5A-B). GO term

297 analysis on significant targets identifies nearly all top predicted pathways relate to deacetylation 
activity, for both selective cytotoxicity and anti-invasion (Fig. 3K, Table S5C-D). These data present herein.

HDAC inhibitors are selectively cytotoxic towards $T S C 2^{-/-}$cells exclusively in hydrogel

culture

We further tested 11 HDAC inhibitors from our compound library at a wider range of concentrations and identified three to be selectively cytotoxic towards $T S C 2^{-}$cells: SAHA (clinically, Vorinostat), SB939 (Pracinostat), and LBH589 (Panobinostat), all of which are panHDAC inhibitors (Fig. 4A). We note the atypical therapeutic dose-response curves and selectivity, demonstrating marginal differences in $\mathrm{IC}_{50}$ per se but substantial variation in maximal toxicity (Fig. 4B). Selective cytotoxicity was largely reversed by co-treatment with rapamycin, suggesting mTORC1-dependency. Importantly, the magnitude of cytotoxic

311 selectivity between WT and $T S C 2^{-/}$cells increased with treatment duration (Fig. S4A). We

312 corroborated selective cell death functionally via clonogenic assays (Fig. S4B). Remarkably,

313 when these HDAC inhibitors were tested with cells cultured on plastic, we did not observe any

314 genotype-selectivity in their cytotoxic profile (Fig. 4A-B). In addition, inhibitor profiles

315 employed in plastic culture did not change in the presence of rapamycin, suggesting a loss of mTORC1-dependency for cytotoxic effects (Fig. 4A-B). While HDAC inhibitors did modulate

317 the proliferation of cells in hydrogel culture, a substantial proliferation blockade was exerted

318 when cells were cultured on plastic, in both genotypes (Fig. S4C). Together, these data indicate a

319 striking difference in cellular responses to HDAC inhibitor treatment while cultured on plastic

320 versus hydrogel. Importantly, HDAC inhibitors only demonstrate mTORC1-dependent selective 
321 toxicity towards $\mathrm{TSC}^{-/}$cells while treated in hydrogel culture. These data are consistent with

322 observation of hydrogel culture potentiating differential mTORC1 signalling between WT and

323 TSC2 $2^{-}$cells (Fig. 2, S2).

HDAC inhibitors induce cell death via apoptosis

We next sought to probe the mode of cell death induced by HDAC inhibitors. Previous mode to be apoptosis. To test this postulation, we employed live cell apoptosis imaging reagents,

331 including cleaved caspase 3 (CASP3) and Annexin V. We validated their activity in our hydrogel

332 culture using staurosporine, a known inducer of apoptosis (Fig. S4D-E). For all three HDAC

333 inhibitors, we observed temporal accumulation of Annexin V and cleaved CASP3 with treatment

334 duration in hydrogel (Fig. 4C-D, S4F). Importantly, we discerned a complete rescue of cell death

335 by co-treatment with the caspase inhibitor Z-VAD (OMe)-FMK (Fig. 4E, S4G). Together, these

336 data demonstrate the employed HDAC inhibitors induce apoptotic cell death in hydrogel culture.

338 HDAC inhibitors are anti-invasive, independent of cytotoxic effects

340 identified and computationally removed $\mathrm{SyTOX}^{+}$cells from invasion calculations (Fig. S5A).

341 We determined all three HDAC inhibitors exhibited a dose-dependent anti-invasion effect on

342 SyTOX ${ }^{-}$cells (Fig. 5A-B). HDAC inhibitors exerted anti-invasive effects on both WT and TSC2-

343 - cells in the presence or absence of rapamycin. (Fig 5A-B, S5B-C). Of note, the effect size was 
344 generally larger in the $T S C 2^{-/-}$cells, and LBH589 demonstrated a trend towards reduced invasion

345 that was not statistically significant. Remarkably, of the 11 HDAC inhibitors we tested, eight

346 demonstrated anti-invasive effects in a dose-dependent manner (Fig. S5D). When aggregated as

347 a class of therapeutics, there is a clear increase in anti-invasive effects with escalating doses,

348 independent of cytotoxicity (Fig. 5C). Together, these data demonstrate HDAC inhibitors are

349 effective anti-invasive agents independent of their cytotoxic profile.

ATP-competitive mTORC1/2 inhibitors potentiate cell invasion

A surprising result of our 3D drug screen is the classification of ATP-competitive

353 mTORC1/2 inhibitors as invasion potentiators (Fig. S3F). We interrogated this further due to its

354 clinical relevance, as ATP-competitive inhibitors are in active development for a wide range of

355 hyperactive mTOR conditions (29). Across a five-point dose-response curve, we observed an

356 increase in invasion from three distinct mTORC1/2 inhibitors, independent of cytotoxic effects

357 (Fig. 5D-E). Invasion potentiation was observed in both WT and TSC2 $^{-/}$cells in the presence or

358 absence of rapamycin (Fig. 5D-E, S5E-F). The extent of invasion potentiation varied across

359 conditions: WT cells exhibited a greater increase in invasion compared to $T S C 2^{-/}$, and the effect

360 was exaggerated in both genotypes by co-treatment with rapamycin (Fig. S5G). Aggregating the

361 effects of all three mTOR inhibitors showed a dose-dependent potentiation of invasion for this

362 class of compounds (Fig. 5F). Together, these data demonstrate ATP-competitive inhibition of

363 mTORC1/2 increases cell invasion. 
We next sought to evaluate the in vivo efficacy of the HDAC inhibitors SAHA, SB939,

and LBH589. Consistent with previous findings, we found that loss of TSC2 alone was mice (Fig. S6A). To avoid immortalization of our cell models- a process which dramatically

371 alters cellular characteristics- we performed a well-established xenotransplantation assay in

372 zebrafish larvae $(31,32)$. In this system, WT or $T S C 2^{-/-}$cells are injected into the hindbrain

373 ventricle of zebrafish larvae 3 days post-fertilization, imaged 1 day post-injection (dpi) to ensure

374 successful engraftment, and then imaged again at 4 dpi to visualize local invasion (Fig. 6A-B).

375 Cells were tracked by their endogenous mCherry expression (14). The optical clarity of this

376 system provides the advantage of enabling isogenic comparisons between WT and TSC2 $2^{-}$

377 human cells in vivo while dynamically tracking cell invasion.

379 found outside the injection site compared to within (Fig. S6B-C). Using this method, we

380 accurately detect near zero invasion scores $1 \mathrm{dpi}$, followed by a substantial increase 4 dpi (Fig.

381 6C). We observed comparable invasion scores between WT and TSC2 $^{-/}$cells which were

382 unaffected by rapamycin treatment, consistent with in vitro data (Fig. 6D). To quantify human

383 cell proliferation and cell death, we digested and pooled whole larvae (15 - 20 per condition)

384 followed by flow cytometry analysis, probing for mCherry and human-specific CASP3.

385 Consistent with xenotransplantation in mice, these cells were not tumorigenic and the rate of

386 clearance outstripped proliferation (Fig. S6D). The number of cells at 4 dpi was comparable

387 between genotypes and unaffected by rapamycin treatment (Fig. 6E). The percentage of CASP3 ${ }^{+}$

388 cells in the mCherry $^{+}$population was $\sim 10 \%$ and equivalent across conditions, similar to baseline

389 cell death rates seen in hydrogel culture (Fig. 3A, 6F). 
391 HDAC inhibitors SAHA and SB939 block cell invasion and selectively eradicate $T S C 2^{-/-}$

392 cells in vivo

393 We next employed our zebrafish xenograft system to assess the efficacy of HDAC

394 inhibitors in vivo. To achieve the highest quality of pre-clinical evidence, experiments were

395 conducted in a randomized, double-blinded, placebo-controlled fashion. We first established

396 dose-toxicity profiles for each HDAC inhibitor: SB939 and LBH589 conferred an IC $_{50}$ of 53.1

$397 \mu \mathrm{M}$ and $6.74 \mu \mathrm{M}$ respectively, while the favourable toxicity profile of SAHA precluded

398 calculation of an $\mathrm{IC}_{50}$ value (Fig. S6E). Of note, in vivo HDAC inhibitor potency correlated with

399 the in vitro cytotoxicity profile. Zebrafish engrafted with either WT or TSC2 $2^{--}$cells were treated

400 with HDAC inhibitors by immersion therapy, in the presence or absence of rapamycin.

401 Importantly, we used the same compound concentration as those employed in vitro, which was

402 well below each compound's $\mathrm{IC}_{50}$ value.

403 After three days of treatment, we observed that SAHA and SB939 exerted a statistically

404 significant anti-invasive effect in the absence of rapamycin, exclusively on the TSC2 $2^{-/}$cells (Fig.

405 6G). SB939 also demonstrated a statistically significant anti-invasive effect in the presence of

406 rapamycin. We note that live cells could not be distinguished from dead or dying cells in this

407 quantification. However, by flow cytometry we observed an increase in the percentage of human

408 TSC2 $^{--}$cells to be CASP3 ${ }^{+}$upon treatment with SAHA and SB939 (Fig. 6H) This effect was

409 abrogated upon combination treatment with rapamycin and was not observed in the human WT

410 cells. Together, these data indicate the HDAC inhibitors SAHA and SB939 exhibit in vivo anti-

411 invasion and selective cytotoxicity effects towards $T S C 2^{-/-}$cells. 


\section{DISCUSSION}

413 Here, we subject novel tissue-engineered models of LAM to a three-dimensional drug

414 screen to detect physiologically relevant therapeutics. We identified HDAC inhibitors as anti-

415 invasive and selectively cytotoxic towards $T S C 2^{-/}$cells, both in vitro and in vivo. In contrast, the

416 gold standard therapeutic agent for LAM patients, rapamycin, did not exhibit any cytotoxic or

417 anti-invasive effects. To our knowledge, this is the first high content compound screen to

418 simultaneously track invasion modulation and cytotoxicity at the single cell level. Importantly,

419 we report the first zebrafish xenotransplantation system using LAM-like cells, extending

420 parametrization of therapeutic effects at the single cell level in vivo while comparing WT versus

421 TSC2 $2^{-/-}$cells.

422 Our research identifies pan-HDAC inhibitors as potential therapeutic candidates for

423 pursuit in LAM patients. Notably, the selective cytotoxic effect towards $T S C 2^{-/}$cells was only

424 observed in hydrogel culture and would have otherwise been missed using standard screening in

425 2D tissue culture plastic (Fig. 4A-B). This finding complements a recent study evidencing

426 therapeutic efficacy of HDAC inhibitors in a $T s c 1^{-/}$-driven mouse model of lymphangiosarcoma

427 (33). HDAC inhibitors present an opportune class of molecules for pursuit due to the wide

428 variety of compounds already approved for clinical use. Indeed, both SAHA and LBH589 are

429 approved for use in cutaneous T cell lymphoma and multiple myeloma, respectively $(34,35)$.

430 The safe-in-human toxicity profile of these compounds will facilitate rapid translation for testing

431 in LAM patients. Importantly, our employed HDAC inhibitors exhibit selective cytotoxicity in

432 an mTORC1-dependent manner, suggesting generalizable efficacy to mTORC1-driven

433 malignancies. Of note, cutaneous T cell lymphoma cells have been observed to exhibit mTORC1

434 hyperactivation compared to matched normal controls (36). 
In this work, we used equivalent HDAC inhibitor concentrations for in vitro and in vivo experiments; these concentrations were well-below dose-limiting toxicities in zebrafish (Fig.

437 S6E). However, a critical outstanding question is whether the concentrations employed are

438 physiologically attainable in humans. Pharmacokinetic studies of SAHA, SB939, and LBH589 in

439 humans have demonstrated micromolar serum concentrations are achievable (37-39). In fact, the

440 original pre-clinical work which formed the foundation for testing SAHA as a treatment in

441 cutaneous T cell lymphoma used the drug in vitro at micromolar concentrations (40). Thus, we

442 anticipate drug concentrations necessary to elicit a therapeutic effect are achievable in patients

443 with LAM. We note that while LBH589 demonstrated a therapeutic effect in vitro, drug efficacy

444 was not maintained upon in vivo testing, possibly due to altered bioavailability in the zebrafish.

445 While SAHA and SB939 demonstrated in vivo efficacy, the majority of parameters assessed

446 demonstrated mTORC1-dependency, similar to in vitro. Many LAM patients are on a chronic

447 regime of rapamycin; thus, it is likely that clinical trials would require short-term withdrawal of

448 rapamycin and acute treatment with HDAC inhibitors to elicit a therapeutic effect. Alternatively,

449 these therapeutics may provide a benefit for LAM patients who are not currently treated with

450 rapamycin, whether due to mild disease, intolerance, or resistance.

451 Our three-dimensional screening approach permitted the identification of many

452 compounds with invasion modulatory capabilities. We encourage further mining of these data to

453 uncover additional novel classes of therapeutics which modulate invasion and/or exert selectivity

454 cytotoxic effects (Table S3B-C). As one example, we identified ATP-competitive inhibitors of

455 mTOR to increase cellular invasion (Fig. 5D-F, S5E-G). This is of clinical significance and

456 requires further investigation, considering this class of therapeutics is undergoing investigation in

457 a broad range of oncogenic conditions (29). 
Throughout our study, we note both genotype (loss of TSC2) and culture substrate (3D hydrogel) contribute to modelling features of LAM. Importantly, hydrogel culture potentiated

460 differential mTORC1-signalling between WT and $T S C 2^{-/-}$cells, reinforcing a physiologically

461 relevant environment in which mTORC1-dependent phenotypes can be identified (Fig 2, S2).

462 However, we also note a variety of LAM features in our cellular models that exist independently

463 from loss of $T S C 2$. For example, cells isolated from both WT and $T S C 2^{-/}$teratomas are equally

464 invasive, present matching ACTA2 $/ \mathrm{PMEL}^{+}$profiles, and secrete similar levels of VEGF-D.

465 Indeed, similar observations of LAM features in WT cells have been noted in a neural crest cell

466 model (14). These data suggest perhaps, while loss of TSC2 is critical for disease pathogenesis,

467 the hallmark features of the putative "LAM cell" may already exist in a physiological, if not

468 transient, context (e.g., during development, injury repair and inflammation). Critical

469 consideration of the cell context is essential, even while employing isogenic comparisons, as

470 different cell types exhibit distinct therapeutic vulnerabilities (14).

471 In summary, we have identified HDAC inhibitors as anti-invasive and selectively

472 cytotoxic towards TSC2 $^{-/-}$cells in vitro and in vivo. While we have investigated three pan-HDAC

473 inhibitors as potential candidates, our data points towards SAHA as the most efficacious against

$474 \mathrm{TSC}^{-/}$cells while possessing the most favourable toxicity profile. On the path towards clinical

475 translation, we anticipate testing of these compounds in diverse disease models. By validating

476 compounds with orthogonal tools and techniques, we may elevate the most promising

477 therapeutic for clinical trials. 


\section{MATERIALS AND METHODS}

\section{Study design}

The objective of this research was to assess the LAM disease modelling capabilities of

481 newly developed tissue-engineered cell models, and subsequently employ these models to

482 identify novel therapeutic compounds. We conducted a 3D drug screen, and based on the

483 acquired data, formulated and tested the following hypothesis: HDAC inhibitors are anti-invasive

484 and selectively cytotoxic towards $\mathrm{TSC}^{-/}$cells. We employed a combination of in vitro and in

485 vivo tools to test this hypothesis. Drug screen data was analyzed in a blinded, unbiased manner,

486 and independently by two different researchers using distinct methods. Animal studies were

487 conducted and analyzed in a double-blinded, randomized, placebo-controlled manner to generate

488 the highest quality pre-clinical evidence. Blinding was achieved by codification of an

489 investigator uninvolved in the experiments performed. A variety of experimental tools were

490 employed to interrogate this hypothesis, described in subsequent Methods and in the

491 Supplementary Materials and Methods. All reagents used and concentrations employed (if

492 relevant) are reported in Supplementary Materials and Methods.

$493 \quad$ Sample sizes for both in vitro and in vivo studies were determined according to field-

494 specific conventions. Power analysis was not employed. Data collection was not stopped

495 prematurely, and every experimental replicate was analyzed. All data points were included in the

496 data presentation; outliers were only excluded if there was definitive empirical evidence of

497 technical error and noted as such in the figure legend. Experiments were repeated at least three

498 times unless otherwise noted, with replicates collected at separate points in time and under

499 independent conditions. RNA-seq data is accessible at the Gene Expression Omnibus (GEO)

500 repository with accession GSE179044. 
Cell derivation and maintenance culture

LAM cell models were established via a previously reported in vivo differentiation

504 protocol of human pluripotent stem cells (23). Briefly, we injected hPSCs into NSG mice to form

505 teratomas, which were then explanted and expanded in smooth muscle-cell enriching conditions

506 (Fig. S1A). We used a previously reported pair of mCherry ${ }^{+}$WT and genome-engineered TSC2 $^{-/-}$

507 hPSCs for establishment of isogenic lines (14). Maintenance cultures were propagated on plastic

508 containing a thin layer of Matrigel at $37^{\circ} \mathrm{C}, 5 \% \mathrm{CO}_{2}$. hPSCs were cultured in Essential 8 medium

509 and passaged in clumps by EDTA incubation, followed by cell scraping and wide-pore pipette

510 transfer. LAM cells were cultured on Matrigel in Medium 231 containing Smooth Muscle

511 Growth Supplement and passaged by $0.05 \%$ Trypsin as single cell suspensions.

\section{Hydrogel culture}

514 Hydrogel culture was conducted according to a previously established protocol (18).

515 Briefly, a hyaluronic acid polymer backbone was derivatized with methylfuran motifs

516 (confirmed by ${ }^{1} \mathrm{H}$ NMR) and conjugated to bismaleimide-terminated vitronectin and collagen-I-

517 derived peptides, synthesized in house. Hydrogel viscoelasticity was increased by incorporation

518 of methylcellulose derivatized with reactive thiol groups. Chemically synthesized hydrogel

519 components were mixed and directly added to culture plates (384-well format) to gel at $37^{\circ} \mathrm{C}$ for

5203 hours. Following gelation, wells were hydrated with PBS and then subjected to three media

521 washes interspaced with incubations at $37^{\circ} \mathrm{C}$ for 45 mins. LAM cells were then dissociated,

522 added to plates containing hydrogel, and centrifuged for $3 \mathrm{~min}$. at $10 \mathrm{~g}$ to achieve immediate

523 contact with the hydrogel. 


\section{Cell treatments and live cell staining}

All compound treatments were conducted for 72 hours unless otherwise stated. See

527 Supplementary Materials and Methods for 3D screen design, implementation, and analysis. Live

528 cell imaging dyes (Hoechst, SyTOX, Annexin V, Cleaved Caspase 3) were incubated for 30 min.

529 prior to imaging. Dyes were added as $10 \mathrm{X}$ concentrates in PBS; Annexin V diluent also

530 contained $2.5 \mathrm{mM} \mathrm{CaCl}_{2}$. To avoid cell detachment in the miniaturized well format, live imaging

531 dyes were not washed prior to imaging; this did not impact image acquisition as dyes are

532 minimally fluorescent unless bound to the target molecule.

\section{Microscopy}

535 We employed a high content imager (Thermo Fisher Scientific, Arrayscan VTI) to

536 acquire multi-well and multi-planar images. Whole-well images (384-well plate format) of cells

537 invading through hydrogel, stained with live cell dyes, were acquired by widefield microscopy

538 with $40 \mu \mathrm{m}$ interval z-stacks. Unstained cells were imaged using a brightfield module. Tiled

539 images of cells grown and stained on plastic were also acquired by high content widefield

540 microscopy. Rodent subcutaneous xenografts were visualized by in vivo imaging (PerkinElmer,

541 IVIS®). Zebrafish larvae xenografts were imaged by epifluorescence widefield microscopy

542 (Zeiss, AxioObserver 7). Image analysis methods are reported in Supplementary Materials and

543 Methods. 
All animal experiments were conducted with approval from the University of Ottawa

547 Animal Care Committee (Protocols \#OHRI1666 and \#CHEOe-3171), in accordance with the

548 Canadian Council on Animal Care Standards and the Province of Ontario's Animals for

549 Research Act. NSG mice (Jackson Laboratory) were maintained in sterile housing conditions and

550 fed autoclaved chow and water ad libitum. Adult casper (41) zebrafish (a gift from Dr. Leonard

551 Zon, Boston Children's Hospital, Boston, MA) were maintained in a recirculating commercial

552 housing system (Aquatic Habitats, now Pentair) at $28^{\circ} \mathrm{C}$ in $14 \mathrm{~h}: 10 \mathrm{~h}$ light:dark conditions in the

553 aquatics facility at the University of Ottawa, Ottawa, ON. Adult casper zebrafish were bred

554 according to standard protocol (42), and embryos were collected and grown in E3 medium (5mM

$\left.555 \mathrm{NaCl}, 0.17 \mathrm{mM} \mathrm{KCl}, 0.33 \mathrm{mM} \mathrm{CaCl}_{2}, 0.33 \mathrm{mM} \mathrm{MgSO}_{4}\right)$ at $28^{\circ} \mathrm{C}$ in $10 \mathrm{~cm}$ Petri dishes until the

556 desired time point. Embryos were cleaned and provided with new media every $24 \mathrm{hrs}$. See

557 Supplementary Materials and Methods for additional experimental details.

Statistical analysis

All figures are presented with individual data points (where graphically appropriate), with

561 measures of central tendency and error to be mean and standard deviation, respectively, unless

562 otherwise stated. Data pre-processing, statistical tests employed, sample number, and measured

563 of central tendency and spread are reported in the figure legends. Two-sided tests were

564 employed, and significance was attributed when $\mathrm{p}<0.05$. All analyses were of data from three

565 independent experiments without removal of statistical outliers. Statistical tests employed were

566 parametric except for analyses of zebrafish invasion data, where a non-normal distribution was

567 observed. Calculation of drug screen statistics (e.g., z-scores, selectivity scores) are described in

568 the Supplementary Materials and Methods. 


\section{LIST OF SUPPLEMENTARY MATERIALS}

570

571 Supplementary Materials and Methods

572

573 Fig. S1. Hydrogel culture of stem cell-derived disease models exhibits features of LAM.

Fig. S2. Hydrogel culture potentiates differential mTORC1-signalling between WT and TSC2 ${ }^{-/-}$

576 cells.

Fig. S3. Three-dimensional drug screen identifies HDAC inhibitors as anti-invasive and

579 selectively cytotoxic towards TSC2 $2^{-/-}$LAM cells.

580

581 Fig. S4. Three safe-in-human HDAC inhibitors induce mTORC1-dependent selective

582 cytotoxicity exclusively in hydrogel culture.

584 Fig. S5. HDAC inhibitors attenuate cell invasion independent of cytotoxicity while mTOR 585 inhibitors potentiate the invasion phenotype.

587 Fig. S6. HDAC inhibitors are anti-invasive and selectively cytotoxic towards TSC2 $2^{-/}$cells 588 xenotransplanted into zebrafish.

590 Table S1. Differential gene expression analysis of bulk RNA-seq data, untreated samples only. 
592 Table S2. GO term enrichment analysis of DEG lists.

593

594 Table S3. Three-dimensional drug screen raw data.

595

596 Table S4. Enrichment results via adaptation of GSEA.

598 Table S5. Elion ${ }^{\mathrm{TM}}$ structure-based compound analysis.

600 Movie S1. Brightfield Z-stack of WT invading through the hydrogel, counterstained with

601 Hoechst.

602

603 Movie S2. Brightfield Z-stack of $T S C 2^{-/}$invading through the hydrogel, counterstained with

604 Hoechst. 


\section{REFERENCES}

1. A. M. Taveira-DaSilva, J. Moss, Clinical features, epidemiology, and therapy of lymphangioleiomyomatosis, Clin Epidemiol 7, 249-257 (2015).

2. J. Moss, N. A. Avila, P. M. Barnes, R. A. Litzenberger, J. Bechtle, P. G. Brooks, C. J. Hedin, Lymphangioleiomyomatosis (LAM) in Patients with Tuberous Sclerosis Complex, Am J Respir Crit Care Med 164, 669-671 (2001).

3. X. Zhe, L. Schuger, Combined Smooth Muscle and Melanocytic Differentiation in correlation, Radiographics 25, 803-828 (2005).

617 5. S. C. Chu, K. Horiba, J. Usuki, N. A. Avila, C. C. Chen, W. D. Travis, V. J. Ferrans, J. Moss, Comprehensive Evaluation of 35 Patients With Lymphangioleiomyomatosis, Chest 115,

6. T. Urban, R. Lazor, J. Lacronique, M. Murris, S. Labrune, D. Valeyre, J. F. Cordier, 78, 321-337 (1999).

7. E. P. Henske, F. X. McCormack, Lymphangioleiomyomatosis - a wolf in sheep's clothing, $J$ Clin Invest 122, 3807-3816 (2012). 
8. A. M. Taveira-DaSilva, O. Hathaway, M. Stylianou, J. Moss, Changes in lung function and chylous effusions in patients with lymphangioleiomyomatosis treated with sirolimus, Ann. Intern. Med. 154, 797-805, W-292-293 (2011).

9. J. Bee, S. Fuller, S. Miller, S. R. Johnson, Lung function response and side effects to rapamycin for lymphangioleiomyomatosis: a prospective national cohort study, Thorax $\mathbf{7 3 ,}$

10. J. Yao, A. M. Taveira-DaSilva, A. M. Jones, P. Julien-Williams, M. Stylianou, J. Moss, Sustained effects of sirolimus on lung function and cystic lung lesions in lymphangioleiomyomatosis, Am. J. Respir. Crit. Care Med. 190, 1273-1282 (2014). Chapman, M. L. Brantly, J. M. Stocks, K. K. Brown, J. P. I. Lynch, H. J. Goldberg, L. R. and Safety of Sirolimus in Lymphangioleiomyomatosis. http://dx.doi.org/10.1056/NEJMoa1100391 (2011), doi:10.1056/NEJMoa1100391.

12. J. J. Bissler, F. X. McCormack, L. R. Young, J. M. Elwing, G. Chuck, J. M. Leonard, V. J.

13. E. A. Goncharova, D. A. Goncharov, A. Eszterhas, D. S. Hunter, M. K. Glassberg, R. S. Yeung, C. L. Walker, D. Noonan, D. J. Kwiatkowski, M. M. Chou, R. A. Panettieri, V. P. 
phosphorylation. A role for the TSC2 tumor suppressor gene in pulmonary lymphangioleiomyomatosis (LAM), J. Biol. Chem. 277, 30958-30967 (2002).

14. S. P. Delaney, L. M. Julian, A. Pietrobon, J. Yockell-Lelièvre, C. Doré, T. T. Wang, V. C. Doyon, A. Raymond, D. A. Patten, A. S. Kristof, M.-E. Harper, H. Sun, W. L. Stanford, Human pluripotent stem cell modeling of tuberous sclerosis complex reveals lineage-specific therapeutic vulnerabilities, bioRxiv , 683359 (2020).

15. D. J. Kwiatkowski, Animal Models of Lymphangioleiomyomatosis (LAM) and Tuberous

16. S. R. Caliari, J. A. Burdick, A practical guide to hydrogels for cell culture, Nature Methods 13, 405-414 (2016).

17. J. A. Burdick, G. D. Prestwich, Hyaluronic Acid Hydrogels for Biomedical Applications, Advanced Materials 23, H41-H56 (2011).

18. R. Y. Tam, J. Yockell-Lelièvre, L. J. Smith, L. M. Julian, A. E. G. Baker, C. Choey, M. S. Model Invasive Lung Diseases Enabling High-Content Drug Screening, Advanced Materials 31, 1806214 (2019).

19. R. Edmondson, J. J. Broglie, A. F. Adcock, L. Yang, Three-dimensional cell culture systems 

(2019).

672 21. C. A. MacRae, R. T. Peterson, Zebrafish as tools for drug discovery, Nature Reviews Drug 673 Discovery 14, 721-731 (2015).

674 22. D. C. Swinney, J. Anthony, How were new medicines discovered?, Nature Reviews Drug Discovery 10, 507-519 (2011). Tam, K. Giannikou, F. McMurray, M. S. Shoichet, M.-E. Harper, E. P. Henske, D. J. Kwiatkowski, T. N. Darling, J. Moss, A. S. Kristof, W. L. Stanford, Human Pluripotent Stem Cell-Derived TSC2-Haploinsufficient Smooth Muscle Cells Recapitulate Features of Lymphangioleiomyomatosis, Cancer Res (2017), doi:10.1158/0008-5472.CAN-17-0925.

24. M. Guo, J. J. Yu, A. K. Perl, K. A. Wikenheiser-Brokamp, M. Riccetti, E. Y. Zhang, P. Sudha, M. Adam, A. Potter, E. J. Kopras, K. Giannikou, S. S. Potter, S. Sherman, S. R. Hammes, D. J. Kwiatkowski, J. A. Whitsett, F. X. McCormack, Y. Xu, Single Cell Transcriptomic Analysis Identifies a Unique Pulmonary Lymphangioleiomyomatosis Cell, American Journal of Respiratory and Critical Care Medicine (2020), doi:10.1164/rccm.201912-2445OC.

25. H. Zhang, G. Cicchetti, H. Onda, H. B. Koon, K. Asrican, N. Bajraszewski, F. Vazquez, C. L. Carpenter, D. J. Kwiatkowski, Loss of Tsc1/Tsc2 activates mTOR and disrupts PI3K-Akt signaling through downregulation of PDGFR, J Clin Invest 112, 1223-1233 (2003).

26. A. Giese, M. A. Loo, N. Tran, D. Haskett, S. W. Coons, M. E. Berens, Dichotomy of astrocytoma migration and proliferation, Int. J. Cancer 67, 275-282 (1996). 
27. A. J. Valvezan, B. D. Manning, Molecular logic of mTORC1 signalling as a metabolic rheostat, Nat Metab 1, 321-333 (2019).

28. D. S. Schrump, Cytotoxicity Mediated by Histone Deacetylase Inhibitors in Cancer Cells: Mechanisms and Potential Clinical Implications, Clin Cancer Res 15, 3947-3957 (2009).

29. A. S. Alzahrani, PI3K/Akt/mTOR inhibitors in cancer: At the bench and bedside, Seminars in Cancer Biology 59, 125-132 (2019).

30. A. L. Fridman, M. A. Tainsky, Critical pathways in cellular senescence and immortalization revealed by gene expression profiling, Oncogene 27, 5975-5987 (2008).

31. B. Adane, G. Alexe, B. K. A. Seong, D. Lu, E. E. Hwang, D. Hnisz, C. A. Lareau, L. Ross,

32. A. M. El-Naggar, C. J. Veinotte, H. Cheng, T. G. P. Grunewald, G. L. Negri, S. P. driven tumours through HDAC inhibition by exploiting innate vulnerability of mTORC1 hyper-activation, British Journal of Cancer , 1-12 (2020). 
34. B. S. Mann, J. R. Johnson, M. H. Cohen, R. Justice, R. Pazdur, FDA approval summary: vorinostat for treatment of advanced primary cutaneous T-cell lymphoma, Oncologist 12, $1247-1252$ (2007).

35. L. A. Raedler, Farydak (Panobinostat): First HDAC Inhibitor Approved for Patients with Relapsed Multiple Myeloma, Am Health Drug Benefits 9, 84-87 (2016).

36. T. E. Witzig, C. Reeder, J. J. Han, B. LaPlant, M. Stenson, H. W. Tun, W. Macon, S. M. Ansell, T. M. Habermann, D. J. Inwards, I. N. Micallef, P. B. Johnston, L. F. Porrata, J. P. Colgan, S. Markovic, G. S. Nowakowski, M. Gupta, The mTORC1 inhibitor everolimus has antitumor activity in vitro and produces tumor responses in patients with relapsed T-cell lymphoma, Blood 126, 328-335 (2015).

37. E. H. Rubin, N. G. B. Agrawal, E. J. Friedman, P. Scott, K. E. Mazina, L. Sun, L. Du, J. L. the Effects of Food and Multiple Dosing on the Pharmacokinetics of Vorinostat Given Orally

38. F. Giles, T. Fischer, J. Cortes, G. Garcia-Manero, J. Beck, F. Ravandi, E. Masson, P. Rae, G.

39. W. P. Yong, B. C. Goh, R. A. Soo, H. C. Toh, K. Ethirajulu, J. Wood, V. Novotny-Diermayr, Inhibitor, in Patients with Refractory Hematologic Malignancies, Clin Cancer Res 12, 4628- 
40. C. Zhang, V. Richon, X. Ni, R. Talpur, M. Duvic, Selective Induction of Apoptosis by Histone Deacetylase Inhibitor SAHA in Cutaneous T-Cell Lymphoma Cells: Relevance to Mechanism of Therapeutic Action, Journal of Investigative Dermatology 125, 1045-1052 (2005).

742 41. R. M. White, A. Sessa, C. Burke, T. Bowman, J. LeBlanc, C. Ceol, C. Bourque, M. Dovey, W. Goessling, C. E. Burns, L. I. Zon, Transparent Adult Zebrafish as a Tool for In Vivo

42. M. Westerfield, THE Zebrafish Book a Guide for the Laboratory Use of Zebrafish Danio*.

746 43. A. Subramanian, P. Tamayo, V. K. Mootha, S. Mukherjee, B. L. Ebert, M. A. Gillette, A.

747 Paulovich, S. L. Pomeroy, T. R. Golub, E. S. Lander, J. P. Mesirov, Gene set enrichment

748 analysis: A knowledge-based approach for interpreting genome-wide expression profiles,

$749 \quad$ Proc. Natl. Acad. Sci. 102, 15545-15550 (2005).

750 44. D. P. Corkery, G. Dellaire, J. N. Berman, Leukaemia xenotransplantation in zebrafish 751 chemotherapy response assay in vivo, Br. J. Haematol. 153, 786-789 (2011).

752 45. H. M, T. C, S. Wl, M. P, Human melanoma cells transplanted into zebrafish proliferate, 753 migrate, produce melanin, form masses and stimulate angiogenesis in zebrafish., 754 Angiogenesis 9, 139-151 (2006). 


\section{ACKNOWLEDGEMENTS}

756 We thank Catherine Lawrence for her inspiration, members of our labs for continued insights,

757 the Ottawa Bioinformatics Core Facility (particularly Christopher Porter) for assistance in

758 processing RNA-seq data, and the Human Pluripotent Stem Cell Core Facility and Rima Al-awar

759 (Ontario Institute for Cancer Research) for the custom designed drug library.

761 Funding:

762 Canadian Institutes for Health Research (CIHR) grant FRN-153188 (WLS)

763 LAM Foundation pilot grant LAM0123P01-17 (WLS)

764 Funds from Green Eggs and LAM (WLS and MSS)

765 CIHR Vanier Canada Graduate Scholarship (AP)

766 CIHR Tier 1 Canada Research Chair Program in Integrative Stem Cell Biology (WLS)

768 Author contributions:

769 Conceptualization: AP, JYL, SPD, RYT, JNB, MSS, WLS

770 Methodology: AP, JYL, NM, LJS, NA, CX, NM, LMJ, RYT, MSS, WLS

771 Investigation: AP, JYL, NM, NA, NM, EL, AC, CD

772 Visualization: AP, JYL

773 Funding acquisition: MSS, WLS

774 Supervision: GM, LMJ, ASK, RYT, JNB, MSS, WLS

775 Writing - original draft: AP, WLS

776 Writing - review \& editing: AP, JYL, NM, LJS, SPD, NA, CX, EL, AC, CD, GM, LMJ, ASK,

777 RYT, JNB, MSS, WLS 
bioRxiv preprint doi: https://doi.org/10.1101/2021.07.03.451004; this version posted July 4, 2021. The copyright holder for this preprint (which

was not certified by peer review) is the author/funder, who has granted bioRxiv a license to display the preprint in perpetuity. It is made available under aCC-BY-NC 4.0 International license.

779 Competing interests: Authors declare that they have no competing interests.

781 Data and materials availability: Processed data are available in the main text and the

782 supplementary materials. Raw RNA-seq data is accessible at the GEO repository with accession

783 GSE179044. Code written in house for data processing is available on request. 


\section{FIGURES}
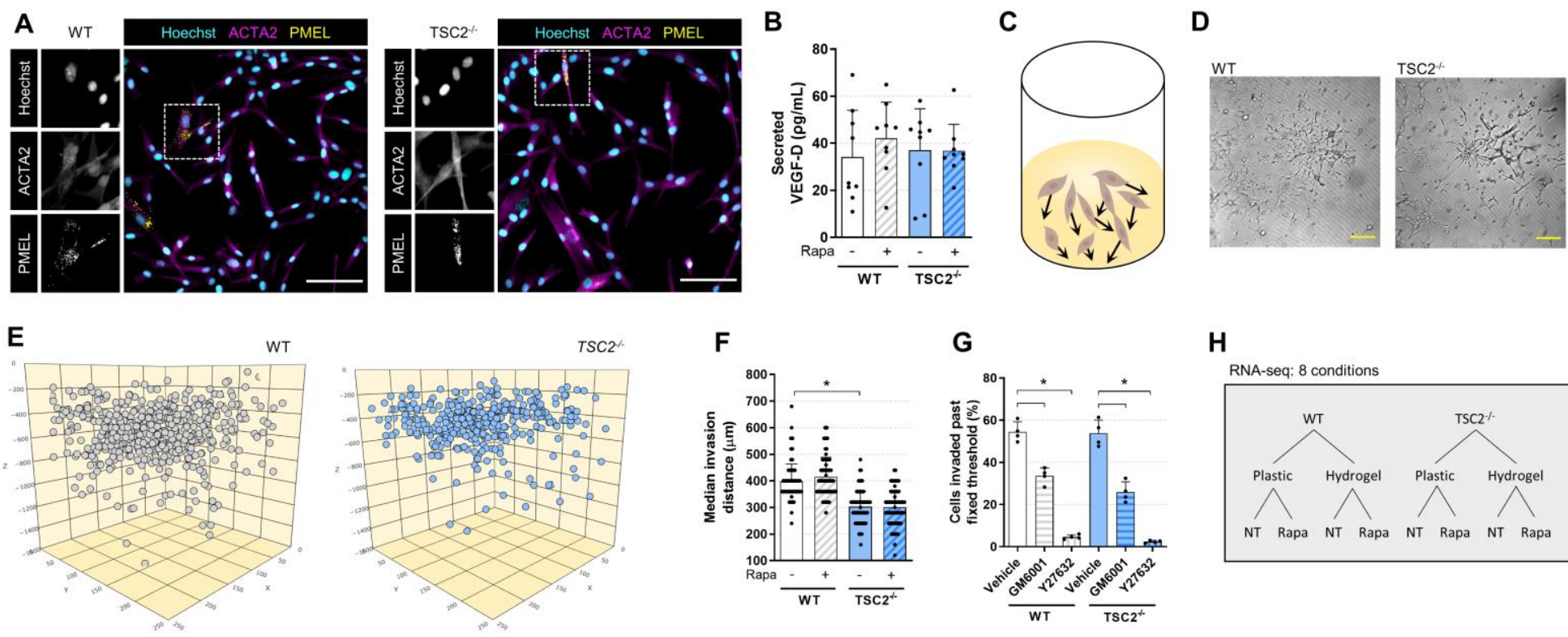

H
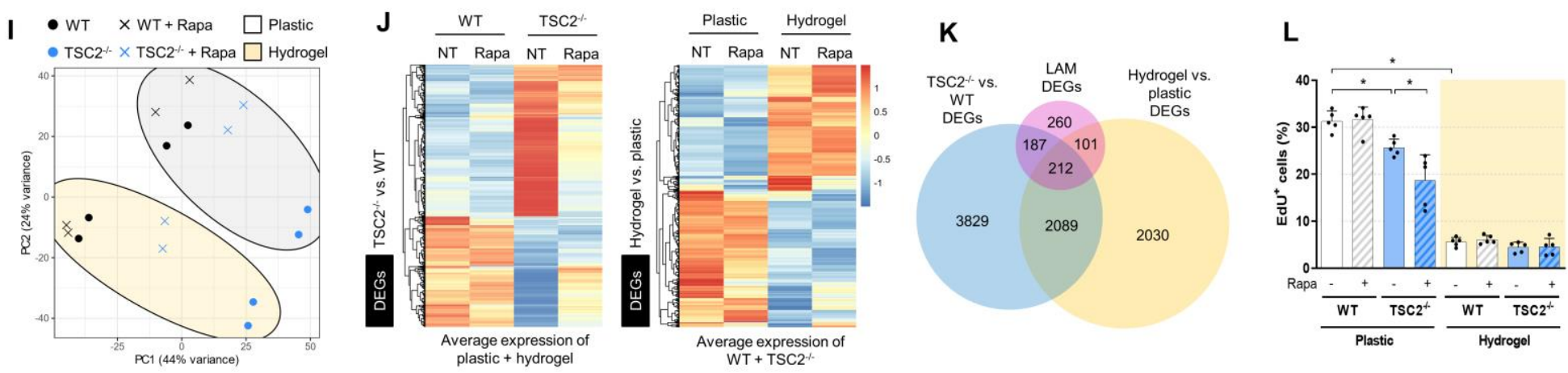

785 Fig. 1. Hydrogel culture of stem cell-derived disease models exhibits features of LAM. (A)

786 Representative immunofluorescence images of $W T$ and $\mathrm{TSC}^{-\%}$ cells. Inset showing punctate

787 PMEL and fibril ACTA2 staining. Scale bars of 100 $\mu$ M. (B) VEGF-D secreted into conditioned

788 media measured by ELISA, following $16 \mathrm{hr}$ incubation in serum-free media $\pm 20 \mathrm{nM}$ rapamycin

789 (mean $\pm \mathrm{SD} ; *=\mathrm{p}<0.05$ by Student $\mathrm{t}$-test; $\mathrm{n}=9-10)$. (C-E) Visualization of LAM cell invasion

790 after three days in hydrogel culture, as (C) a schematic, (D) brightfield image of single Z plane,

791 scale bars of $250 \mu \mathrm{m}$, and (E) computational reconstruction of cellular spatial positions. (F)

792 Median invasion distance of cellular populations plated on the hydrogel and cultured for three

793 days $\pm 20 \mathrm{nM}$ rapamycin (mean $\pm \mathrm{SD} ; *=\mathrm{p}<0.05$ by Student $\mathrm{t}$-test; $\mathrm{n}=124$ ). (G) Percentage of 
794 cells invaded past fixed threshold set by median invasion distance of genotype-matched vehicle

795 control. Cells were cultured and treated for three days (10 $\mu$ M GM6001, a pan-MMP inhibitor,

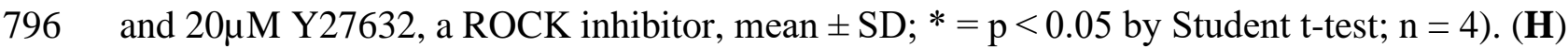

797 Schematic of the sample conditions tested in the bulk RNA-seq experiment. NT $=$ no treatment,

798 Rapa = rapamycin treatment (20nM, 72 hrs). (I) Principal components analysis (PCA) of bulk

799 RNA-seq samples. (J) Heatmap and hierarchal clustering of differentially expressed genes

800 (DEGs) between TSC2 $2^{-/}$and WT samples, and between hydrogel and plastic samples, while

801 controlling for the reciprocal covariate. Left panel: transcript expression for plastic and hydrogel

802 cultures were averaged. Right panel: transcript expression for WT and $T S C 2^{-/}$samples were

803 averaged. DEG analysis was performed with no treatment samples; genes noted as differentially

804 expressed if FDR $<0.05$ and $\left|\log _{2} \mathrm{FC}\right|>1$. (K) Overlap in DEG between genotype and ECM

805 gene lists and LAM cell signature gene list (24). Genes noted as DE if FDR < 0.05. (L)

806 Percentage of $\mathrm{EdU}^{+}$(proliferating) cells from 3-hour pulse $(5 \mu \mathrm{M})$, after three days cultured on

807 plastic or hydrogel $\pm 20 \mathrm{nM}$ rapamycin $($ mean $\pm \mathrm{SD} ; *=\mathrm{p}<0.05$ by Student $\mathrm{t}$-test; $\mathrm{n}=5)$. 

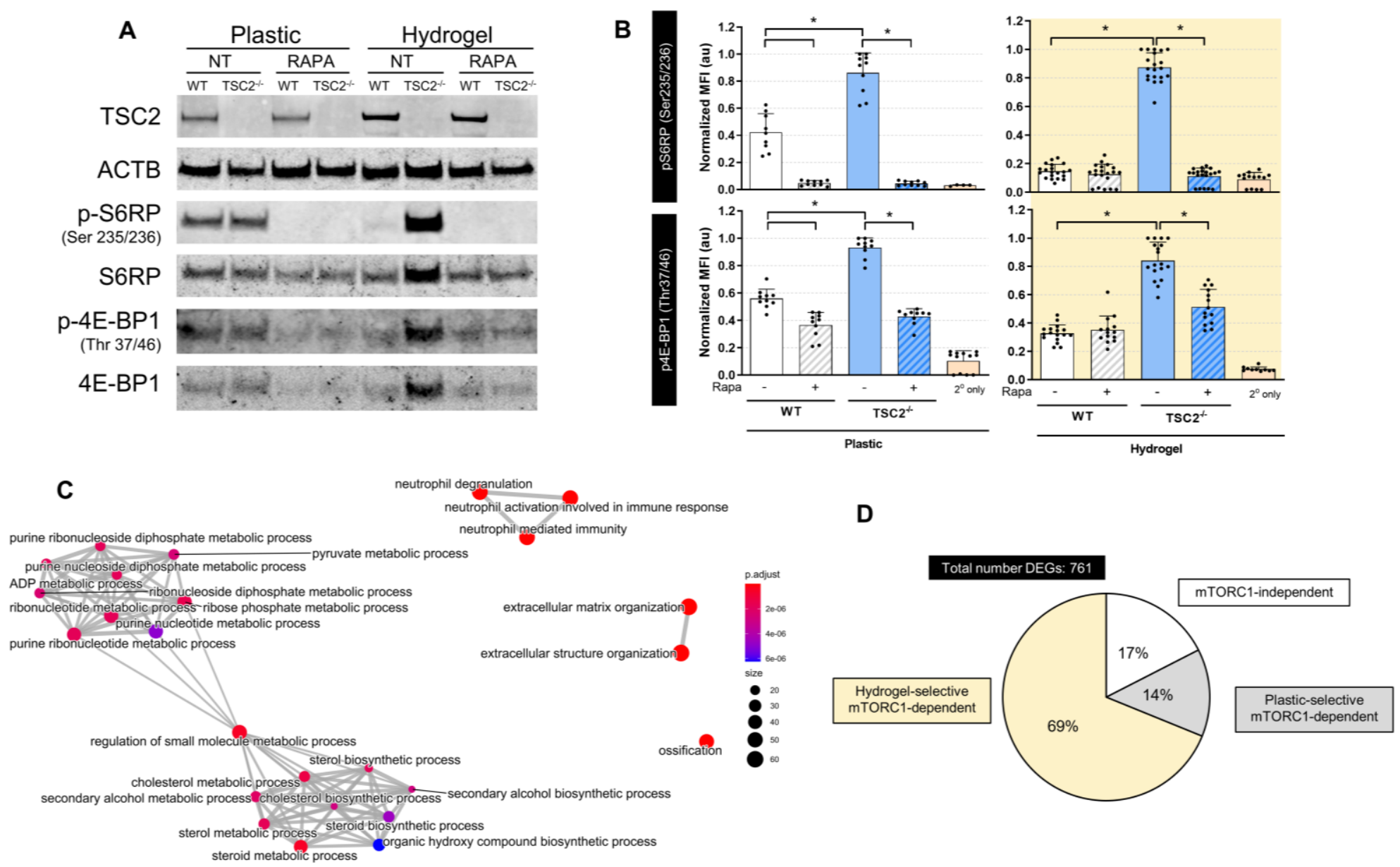

\section{Fig. 2. Hydrogel culture potentiates differential mTORC1-signalling between WT and}

809 TSC2 ${ }^{-/-}$cells. (A) Low input Western blot of protein collected from cells cultured for three days

810 on plastic or hydrogel $\pm 20 \mathrm{nM}$ rapamycin. $\mathrm{NT}=$ no treatment, Rapa $=$ rapamycin treatment. $(\mathbf{B})$

811 Quantification of immunofluorescence values reported in normalized (scaled by replicate

812 maximum value) mean fluorescence intensity. Each point indicates the mean fluorescence

813 intensity from a well of cells cultured on hydrogel or plastic for three days $\pm 20 \mathrm{nM}$ rapamycin.

814 Secondary only values are determined from wells probed with fluorescent secondary antibody

815 only (mean $\pm \mathrm{SD} ; *=\mathrm{p}<0.05$ by Student $\mathrm{t}$-test; $\mathrm{n}=9$-20). (C) Network analysis of GO terms

816 enriched in the list of DEGs found significant $($ FDR $<0.05)$ in the interaction between genotype

817 and culture substrate. The 25 most significantly enriched terms are plotted. (D) Classification of 
bioRxiv preprint doi: https://doi.org/10.1101/2021.07.03.451004; this version posted July 4, 2021. The copyright holder for this preprint (which was not certified by peer review) is the author/funder, who has granted bioRxiv a license to display the preprint in perpetuity. It is made available under aCC-BY-NC 4.0 International license.

818 DEGs according to pattern of expression across genotypes, ECM condition, and in the presence

819 or absence of rapamycin. Gene clusters and classification scheme shown in Fig. S2B-C). 


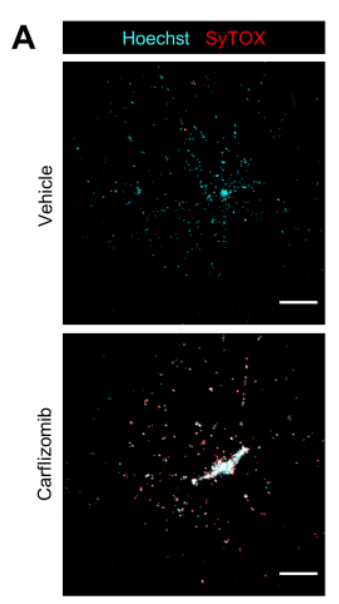

F

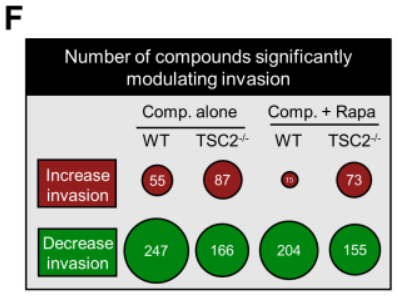

G
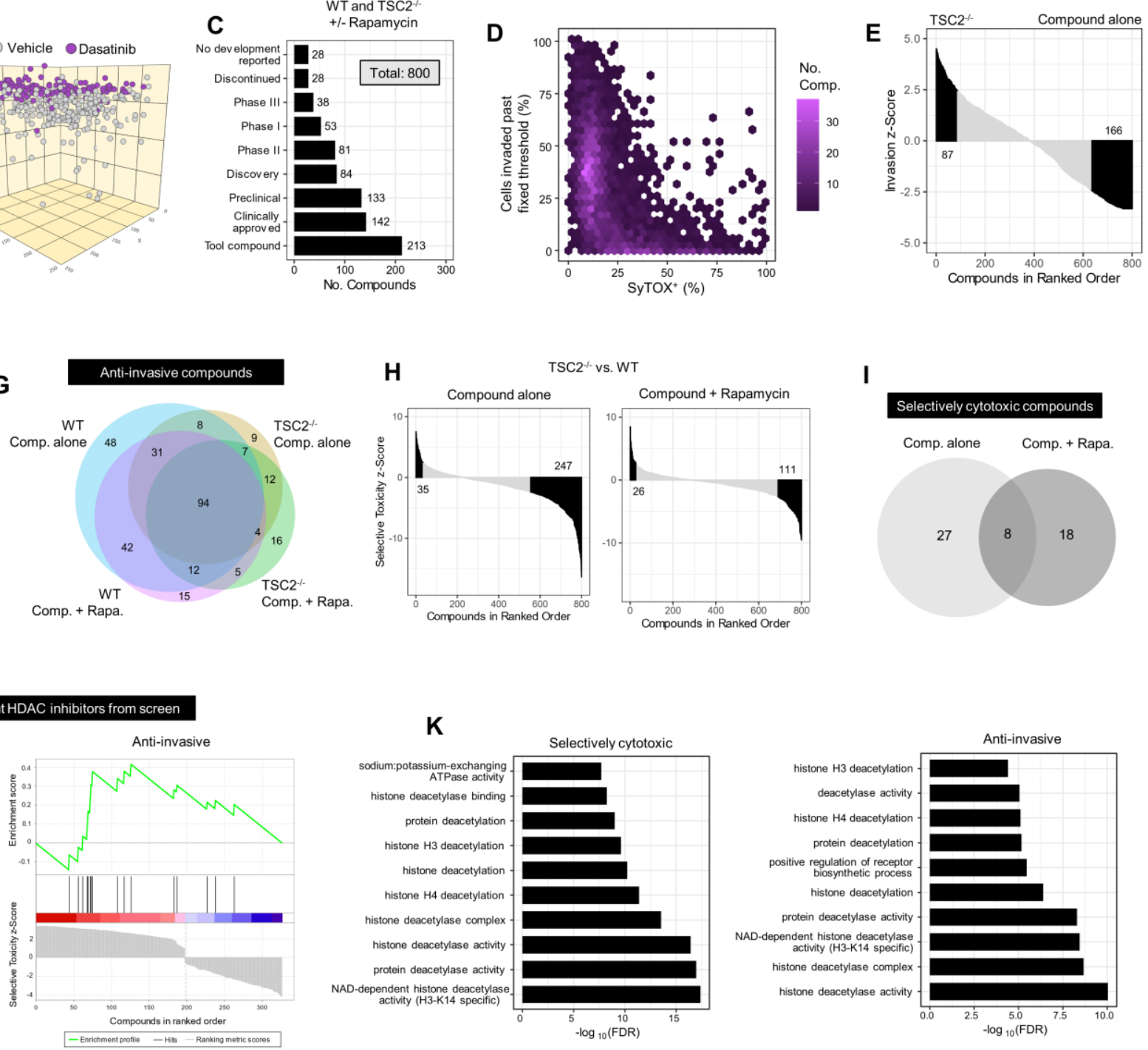

J
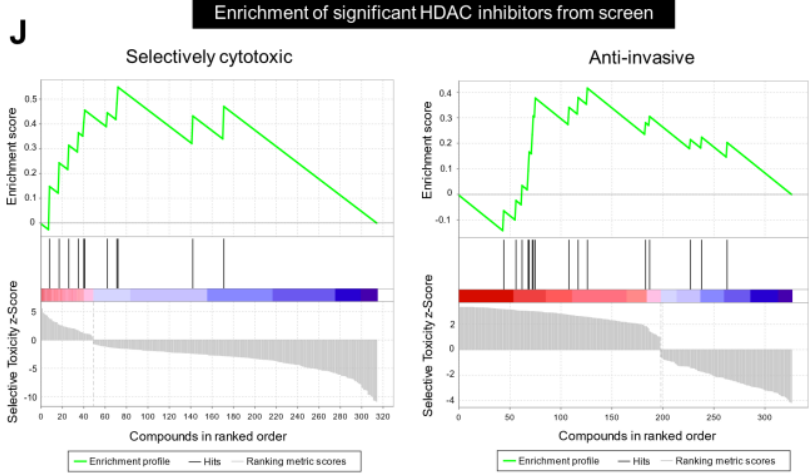

I

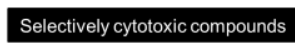

Comp. alone Comp. + Rapa.

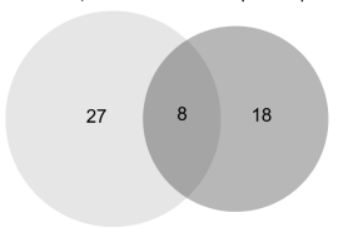

820 Fig. 3. Three-dimensional drug screen identifies HDAC inhibitors as anti-invasive and

821 selectively cytotoxic towards TSC2 ${ }^{-/-}$LAM cells. (A) Representative maximum intensity

822 projection image of $T S C 2^{-/}$cells in hydrogel culture for three days $\pm 200 \mathrm{nM}$ carfilzomib. Scale

823 bars of $250 \mu \mathrm{m}$. (B) Computational reconstruction of cellular spatial positions following three-

824 day hydrogel culture of $T S C 2^{-/}$cells $\pm 40 \mathrm{nM}$ dasatinib. Note that treated and untreated were in

825 separate wells; cells were plotted in the same volume for ease of visualizing relative distances

826 travelled. (C) Highest development status reported for the 800 compounds contained in the

827 curated kinase inhibitor and tool compound libraries. A 3D drug screen was conducted on WT 
and $T S C 2^{-/}$cells following three-day treatment with $5 \mu \mathrm{M}$ compounds $\pm 20 \mathrm{nM}$ rapamycin. (D)

829 Compound invasion modulation plotted against cytotoxicity, aggregating results across genotype

830 and rapamycin treatment. Fixed invasion threshold determined by median invasion distance of

831 untreated controls. Hexagonal plot employed to demonstrate compound densities. (E) Waterfall

832 plot of compound invasion z-scores in ranked order; positive values indicate invasion

833 potentiation, while negative values indicate invasion attenuation. Compounds conferring

834 statistically significant invasion modulation highlighted in black. Data presented for $T S C 2^{-/}$, no

835 rapamycin treatment condition. (F) Number of compounds significantly modulating invasion

836 (potentiating or attenuating) for each genotype in the presence of absence of 20nM rapamycin.

837 Bubble area proportional to number of statistically significant targets. (G) Overlap of compounds

838 identified as anti-invasive in each listed condition. (H) Waterfall plots of compound selective

839 toxicity z-scores in ranked order; positive values indicate increased cytotoxicity towards $T S C 2^{-/-}$

840 cells, negative values indicate increased cytotoxicity towards WT cells. Compounds conferring

841 statistically significant selective cytotoxicity highlighted in black. (I) Overlap of compounds

842 identified to be selectivity cytotoxic towards $T S C 2^{-/-}$cells, with or without $20 \mathrm{nM}$ rapamycin. (J)

843 Enrichment plot for compounds annotated to target HDACs, derived from an adapted

844 implementation of GSEA. Hits (black vertical lines) in the red region indicate compounds with a

845 favourable effect, hits in the blue region indicate compounds with an undesirable effect. (K) Top

84610 most statistically significant GO terms. Analysis performed using targets identified as

847 statistically significantly enriched in screen data by Elion ${ }^{\mathrm{TM}}$ algorithm. 
A

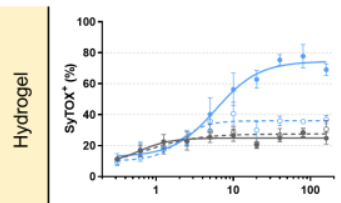

焉

$\rightarrow W T \quad \rightarrow T S C 2^{-} \quad-O-W T+$ Rapa
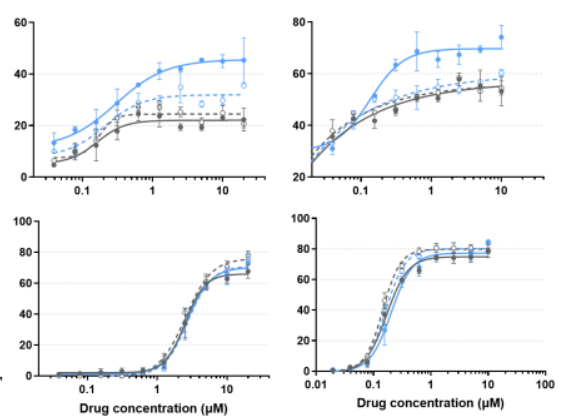

C

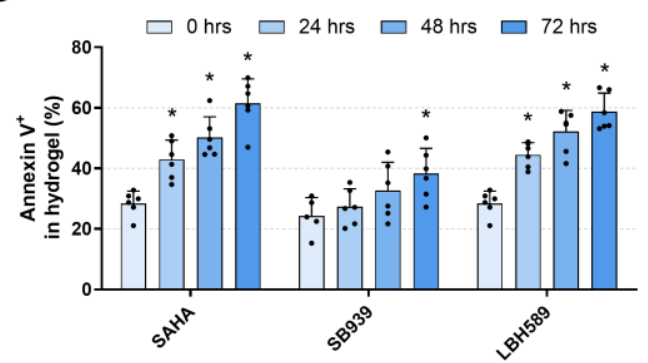

D

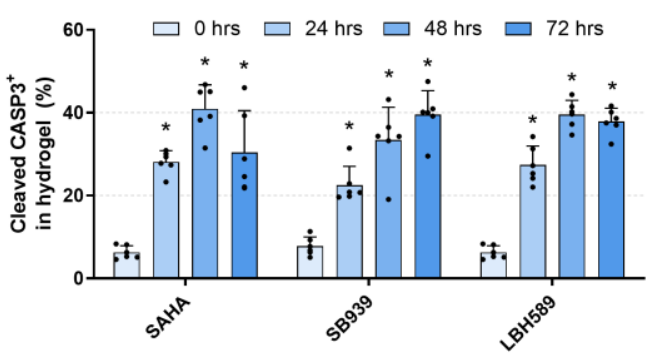

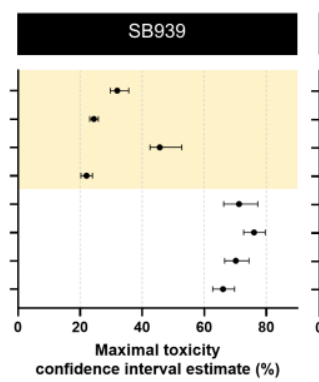

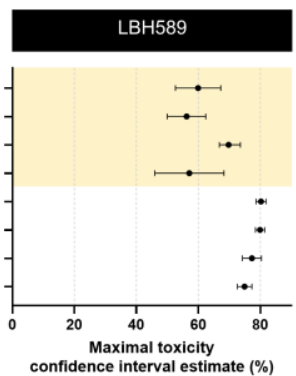

E

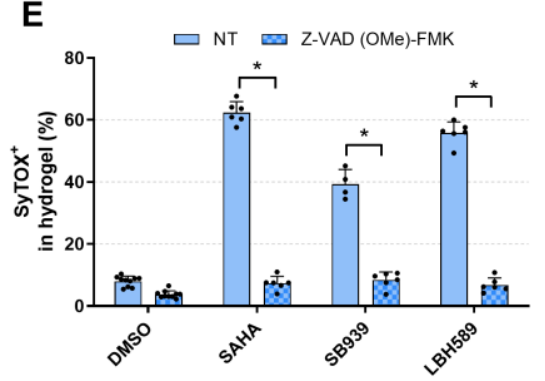

848 Fig. 4. Three safe-in-human HDAC inhibitors induce mTORC1-dependent selective

849 cytotoxicity exclusively in hydrogel culture. (A) Dose-response cytotoxicity curves of cells

850 treated with the indicated HDAC inhibitor for three days while cultured on plastic or hydrogel \pm

851 20nM rapamycin. Data fit via four-parameter logistic regression (mean $\pm \mathrm{SD} ; \mathrm{n}=3)$. (B)

852 Confidence intervals of HDAC inhibitor maximal toxicity, estimated by four-parameter logistic

853 regression models generated in A. (C-D) Quantification of $T S C 2^{-/}$cells positive for (C) Annexin

854 V or (D) cleaved caspase 3, following three-day treatment with HDAC inhibitors in hydrogel

$855(20 \mu \mathrm{M}$ SAHA, $5 \mu \mathrm{M}$ SB939, and $1 \mu \mathrm{M}$ LHB589, mean \pm SD; $*=p<0.05$ by ANOVA with

856 Dunnett post-hoc comparison to $0 \mathrm{hrs} ; \mathrm{n}=6)$. (E) Quantification of $T S C 2^{-/}$cells positive for

857 SyTOX following three-day HDAC inhibitor treatment (20 $\mu \mathrm{M}$ SAHA, $5 \mu \mathrm{M}$ SB939, and $1 \mu \mathrm{M}$

858 LHB589) in hydrogel $\pm 25 \mu \mathrm{M}$ Z-VAD $(\mathrm{OMe})-\mathrm{FMK}($ mean $\pm \mathrm{SD} ; *=\mathrm{p}<0.05$ by Student t-test;

$859 \mathrm{n}=4-6)$. 

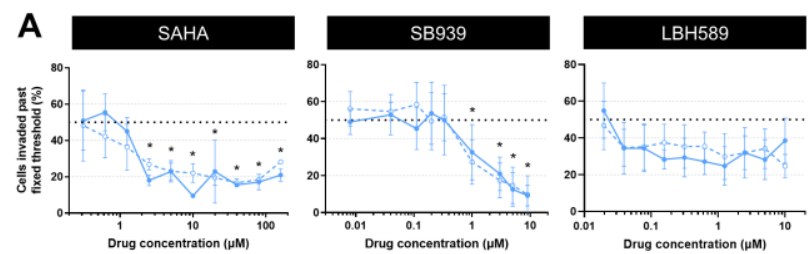

B
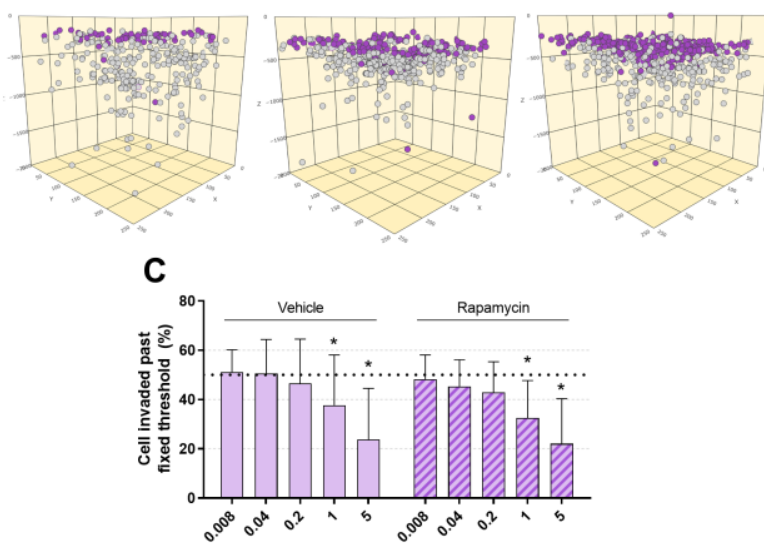

HDACi concentration $(\mu \mathrm{M})$
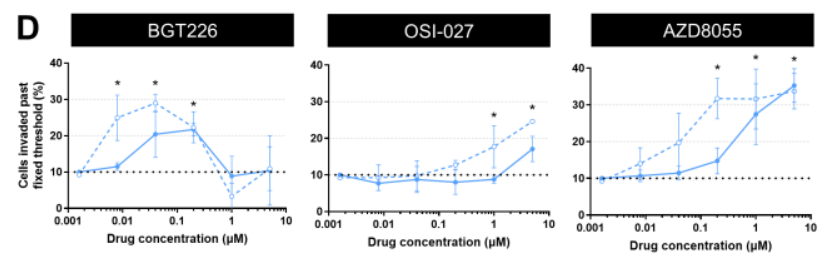

E
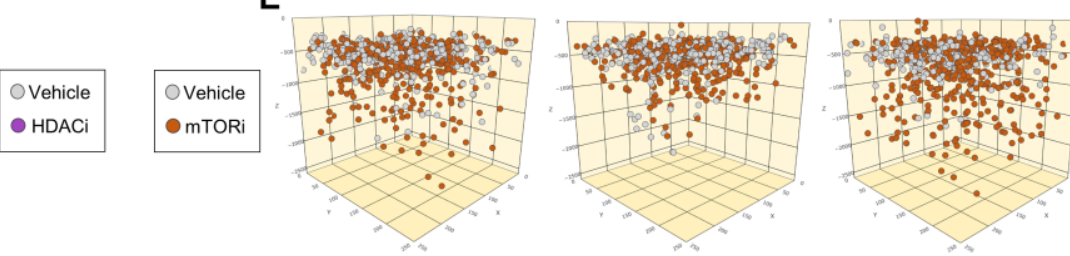

$\mathbf{F}$

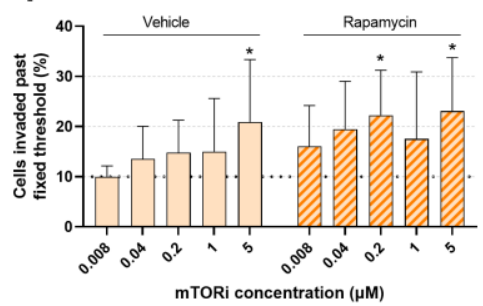

Fig. 5. HDAC inhibitors attenuate cell invasion independent of cytotoxicity while mTOR

861 inhibitors potentiate the invasion phenotype. (A) Live $T S C 2^{-/}$cells invaded past fixed

862 threshold set by median invasion distance of vehicle control, upon three-day HDAC inhibitor

863 treatment $\pm 20 \mathrm{nM}$ rapamycin (mean $\pm \mathrm{SD} ;{ }^{*}=\mathrm{p}<0.05$ by ANOVA with Dunnett post-hoc

864 comparison to untreated; $n=3$ ). (B) Computational reconstruction of live cell spatial positions

865 upon three-day hydrogel culture of $T S C 2^{-/-}$cells \pm HDAC inhibitor (HDACi) treatment $(5 \mu \mathrm{M}$

866 SAHA, $5 \mu \mathrm{M}$ SB939, $1 \mu \mathrm{M}$ LBH589). Note that treated and untreated cells were in separate

867 wells; cells were plotted in the same volume for ease of visualizing relative distances travelled.

868 (C) Aggregated effect of 11 HDAC inhibitors on $T S C 2^{-/-}$live cell invasion $\pm 20 \mathrm{nM}$ rapamycin.

869 Fixed threshold set by median invasion distance of vehicle control (mean $\pm \mathrm{SD} ; *=\mathrm{p}<0.05$ by

870 ANOVA with Dunnett post-hoc comparison to untreated; $\mathrm{n}=33$ via 11 HDACi, $\mathrm{n}=3$ each). (D)

871 Live $T S C 2^{-/}$cells invaded past fixed threshold set by $90^{\text {th }}$ percentile invasion distance of vehicle

872 control, upon three-day mTOR inhibitor treatment $\pm 20 \mathrm{nM}$ rapamycin $($ mean $\pm \mathrm{SD} ; *=\mathrm{p}<0.05$

873 by ANOVA with Dunnett post-hoc comparison to untreated; $\mathrm{n}=3$ ). (E) Computational 
874 reconstruction of live cell spatial positions upon three-day hydrogel culture of $T S C 2^{-/} \pm \mathrm{mTOR}$

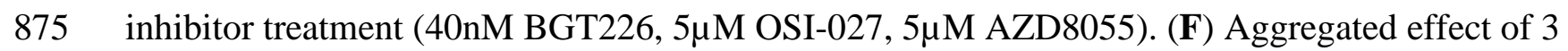

876 mTOR inhibitors (mTORi) on $T S C 2^{-/-}$live cell invasion $\pm 20 \mathrm{nM}$ rapamycin. Fixed threshold set

877 by $90^{\text {th }}$ percentile invasion distance of vehicle control (mean $\pm \mathrm{SD} ; *=\mathrm{p}<0.05$ by ANOVA with

878 Dunnett post-hoc comparison to untreated; $\mathrm{n}=9$ via 3 mTORi, $\mathrm{n}=3$ each). 
A
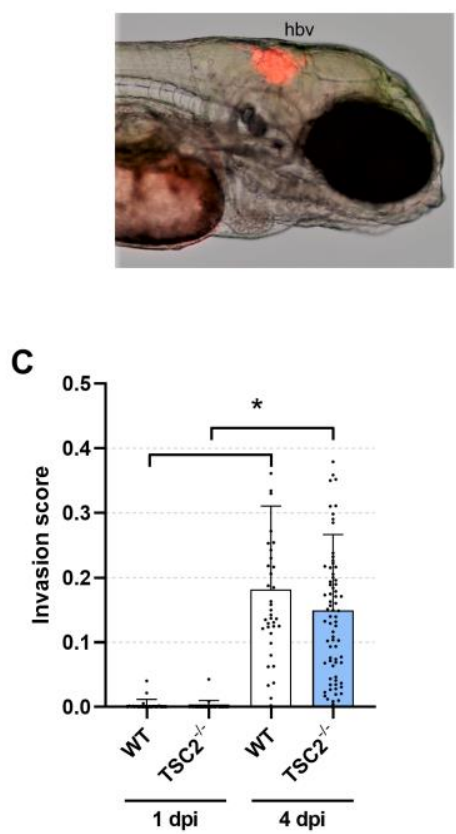

B

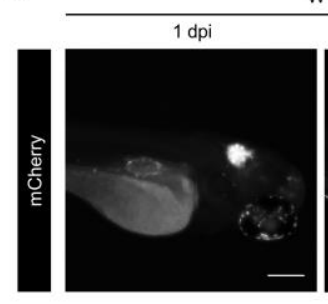

D

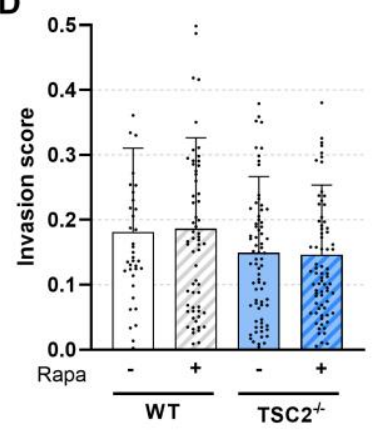

E

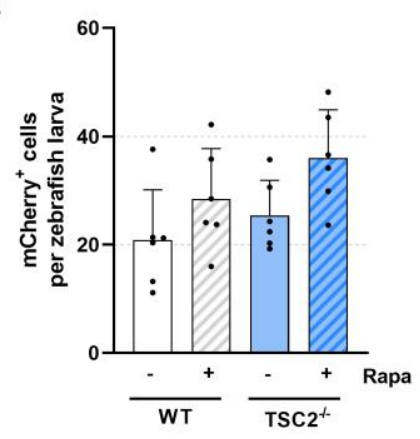

$\mathbf{F}$

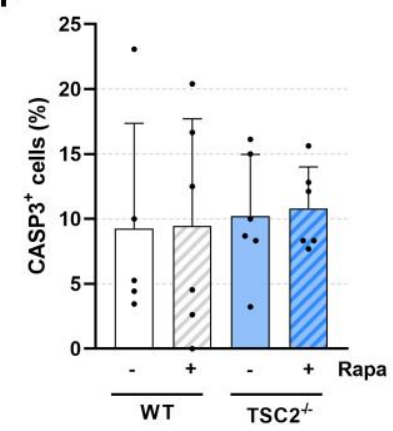

G

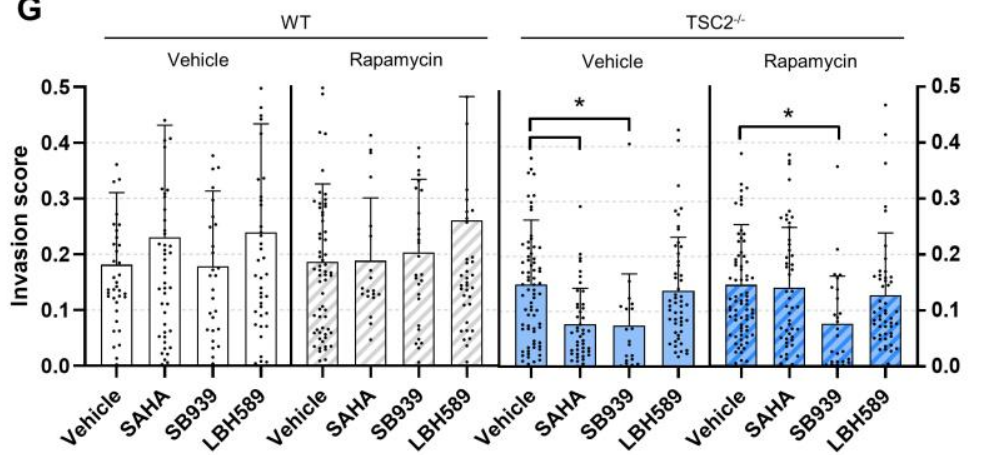

H

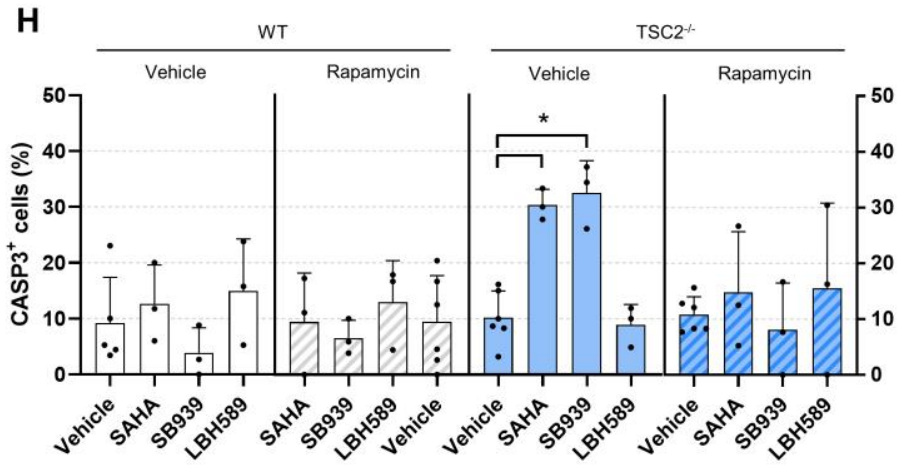

879 Fig. 6. HDAC inhibitors are anti-invasive and selectively cytotoxic towards TSC2 ${ }^{-/-}$cells

880 xenotransplanted into zebrafish. (A) Representative phase contrast image of 1 day post-

881 injection (dpi) zebrafish larvae injected with $\mathrm{TSC}^{-/} \mathrm{mCherry}^{+}$cells into the hindbrain ventricle

882 (hbv). (B) Representative images of zebrafish larvae injected with mCherry ${ }^{+}$WT or TSC2 $2^{-/}$cells

883 into the hbv. Fish were imaged 1 and 4 dpi. Scale bars of $200 \mu \mathrm{m}$. (C-D) Quantification of cell

884 invasion using automated invasion analysis. Images analyzed in (D) were taken 4 dpi following

885 three-day treatment $\pm 20 \mathrm{nM}$ rapamycin $($ mean $\pm \mathrm{SD} ; *=\mathrm{p}<0.05$ by Mann-Whitney $\mathrm{U}$ test; $\mathrm{n}=$

88638 - 73). (E) Number of mCherry ${ }^{+}$cells detected per zebrafish following whole larvae

887 dissociation at 4 dpi and analysis by flow cytometry. Samples were treated for three days \pm 
$88820 \mathrm{nM}$ rapamycin. Each replicate is a pool of $15-20$ zebrafish larvae (mean $\pm \mathrm{SD} ; *=\mathrm{p}<0.05$

by Student t-test; $n=6)$. (F) Percentage of CASP3 ${ }^{+}$cells in the mCherry $^{+}$population from whole

890 larvae dissociation at $4 \mathrm{dpi}$, following 3-day treatment $\pm 20 \mathrm{nM}$ rapamycin. Each replicate is a

891 pool of $15-20$ zebrafish larvae (mean $\pm \mathrm{SD} ; *=\mathrm{p}<0.05$ by Student t-test; $\mathrm{n}=5-6) .(\mathrm{G}-\mathrm{H})$

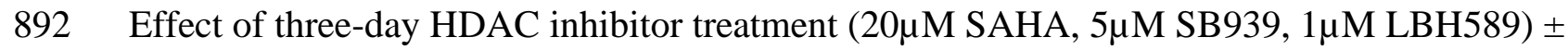

893 20nM rapamycin. (G) Invasion scores calculated on images acquired 4dpi (mean $\pm \mathrm{SD} ; *=$

$894 \mathrm{p}<0.05$ by the Kruskal-Wallis test with Dunn's post-hoc comparison to vehicle treated; $\mathrm{n}=27-$

895 73). (H) Percentage of $\mathrm{CASP}^{+}$cells in the $\mathrm{mCherry}^{+}$population from whole larvae dissociation

896 at 4 dpi. Each replicate is a pool of $15-20$ zebrafish larvae (mean $\pm \mathrm{SD} ;{ }^{*}=\mathrm{p}<0.05$ by

897 ANOVA with Dunnett post-hoc comparison to vehicle treated; $n=3-6)$. Not all outliers in (C-

898 D) and $(\mathrm{G})$ are visualized due to trimmed axes (although outliers were included in mean $\pm \mathrm{SD}$

899 and the statistical calculation). 


\section{Three-dimensional drug screen identifies HDAC inhibitors as therapeutic agents in mTORC1-driven lymphangioleiomyomatosis}

Authors: Adam Pietrobon ${ }^{1,2,3}$, Julien Yockell-Lelièvre ${ }^{1,3}$, Nicole Melong ${ }^{4}$, Laura J. Smith ${ }^{5,6,7}$, Sean P. Delaney ${ }^{1,2,3}$, Nadine Azzam ${ }^{4}$, Chang Xue ${ }^{6,7}$, Nishanth Merwin ${ }^{8}$, Eric Lian ${ }^{1,2,3}$, Alberto Camacho-Magallanes $^{1,2,3}$, Carole Doré ${ }^{1}$, Gabriel Musso ${ }^{8}$, Lisa M. Julian ${ }^{9}$, Arnold S. Kristof ${ }^{10}$, Roger Y. Tam ${ }^{11}$, Jason N. Berman ${ }^{2,4}$, Molly S. Shoichet ${ }^{5,6,7,12}$, William L. Stanford ${ }^{1,2,3 *}$

\section{Affiliations:}

${ }^{1}$ The Sprott Centre for Stem Cell Research, Regenerative Medicine Program, Ottawa Hospital Research Institute; Ottawa, Canada.

${ }^{2}$ Department of Cellular and Molecular Medicine, University of Ottawa; Ottawa, Canada.

${ }^{3}$ Ottawa Institute of Systems Biology; Ottawa, Canada.

${ }^{4}$ Department of Pediatrics, CHEO Research Institute; Ottawa, Canada.

${ }^{5}$ Department of Chemical Engineering and Applied Chemistry, University of Toronto; Toronto, Canada.

${ }^{6}$ Institute for Biomaterials and Biomedical Engineering, University of Toronto; Toronto, Canada.

${ }^{7}$ The Donnelly Centre for Cellular and Biomolecular Research; Toronto, Canada.

${ }^{8}$ BioSymetrics, Inc; Toronto, Canada.

${ }^{9}$ Centre for Cell Biology, Development, and Disease, Department of Biological Sciences, Simon Fraser University; Burnaby, Canada.

${ }^{10}$ Meakins-Christie Laboratories and Translational Research in Respiratory Diseases Program, Research Institute of the McGill University Health Centre, Faculty of Medicine, Departments of Medicine and Critical Care; Montreal, Canada.

${ }^{11}$ Centre for Biologics Evaluation, Biologic and Radiopharmaceutical Drugs Directorate, Health Canada; Ottawa, Canada.

${ }^{12}$ Department of Chemistry, University of Toronto; Toronto, Canada.

* Corresponding author

Dr. William L. Stanford, Ph.D.

The Ottawa Hospital, 501 Smyth Rd, Box 511, CCW 5206c

Ottawa, ON K1H 8L6, Canada

wstanford@ohri.ca

One Sentence Summary: We performed a drug screen in 3D and discovered HDAC inhibitors exhibit therapeutic efficacy in models of the lung disease lymphangioleiomyomatosis. 


\section{SUPPLEMENTARY MATERIALS AND METHODS}

\section{Study reagents and resources}

941 Please see below (Table 1) for a list of key reagents and resources used in this study.

942

943 Table 1: List of key reagents and resources used in this study.

\begin{tabular}{|c|c|c|}
\hline REAGENTS and RESOURCES & SOURCE & IDENTIFIER \\
\hline \multicolumn{3}{|l|}{ Antibodies } \\
\hline PMEL & Thermo Fisher Scientific & MA5-13232 \\
\hline ACTA2 & Abcam & ab5694 \\
\hline mCherry & Abcam & ab167453 \\
\hline pS6RP (Ser 235/236) & CST & 2211 \\
\hline p4E-BP1(Thr 37/46) & CST & 2855 \\
\hline S6RP & CST & 2317 \\
\hline 4E-BP1 & Thermo Fisher Scientific & $\mathrm{AHO} 1382$ \\
\hline TSC2 & CST & 4308 \\
\hline ACTB & CST & 370 \\
\hline Cleaved CASP3 & CST & 9661 \\
\hline mCherry & Thermo Fisher Scientific & $\mathrm{M} 11217$ \\
\hline AlexaFluor 488 Goat Anti-Rabbit lgG $(\mathrm{H}+\mathrm{L})$ & Thermo Fisher Scientific & A11034 \\
\hline AlexaFluor 488 Goat Anti-Mouse lgG $(\mathrm{H}+\mathrm{L})$ & Thermo Fisher Scientific & A11001 \\
\hline AlexaFluor 555 Goat Anti-Rabbit lgG $(\mathrm{H}+\mathrm{L})$ & Thermo Fisher Scientific & A21428 \\
\hline AlexaFluor 555 Goat Anti-Mouse lgG (H+L) & Thermo Fisher Scientific & A32727 \\
\hline AlexaFluor 647 Goat Anti-Rabbit IgG $(\mathrm{H}+\mathrm{L})$ & Thermo Fisher Scientific & A21245 \\
\hline AlexaFluor 647 Goat Anti-Mouse lgG $(\mathrm{H}+\mathrm{L})$ & Thermo Fisher Scientific & $\mathrm{A} 21235$ \\
\hline AlexaFluor 680 Goat Anti-Rabbit IgG $(\mathrm{H}+\mathrm{L})$ & Thermo Fisher Scientific & A21076 \\
\hline AlexaFluor 680 Goat Anti-Mouse lgG $(\mathrm{H}+\mathrm{L})$ & Thermo Fisher Scientific & A28183 \\
\hline DyLight 800 Goat Anti-Rabbit IgG $(\mathrm{H}+\mathrm{L})$ & Thermo Fisher Scientific & SA535571 \\
\hline DyLight 800 Goat Anti-Mouse IgG $(\mathrm{H}+\mathrm{L})$ & Thermo Fisher Scientific & SA535521 \\
\hline \multicolumn{3}{|l|}{ Live cell imaging dyes } \\
\hline Hoechst 33342 & Thermo Fisher Scientific & $\mathrm{H} 3570$ \\
\hline SyTOX Green & Thermo Fisher Scientific & S7020 \\
\hline NucView 488 Caspase-3 Enzyme Substrate & Biotium & 10402 \\
\hline Annexin V & Biotium & 29003 \\
\hline \multicolumn{3}{|l|}{ Small molecules } \\
\hline Kinase Inhibitor library & $\begin{array}{l}\text { Ontario Institute for Cancer } \\
\text { Research }\end{array}$ & $\bar{N} / \mathrm{A}$ \\
\hline Tool Compound library & $\begin{array}{l}\text { Ontario Institute for Cancer } \\
\text { Research }\end{array}$ & $\mathrm{N} / \mathrm{A}$ \\
\hline Rapamycin & MilliporeSigma & 553211 \\
\hline Staurosporine & AdooQ Bioscience & A10864 \\
\hline SAHA & AdooQ Bioscience & A10979 \\
\hline SB939 & AdooQ Bioscience & A10830 \\
\hline LBH589 & AdooQ Bioscience & A10518 \\
\hline BGT226 & AdooQ Bioscience & A11162 \\
\hline OSI-027 & Toronto Research Chemicals & A611910 \\
\hline AZD8055 & AdooQ Bioscience & A10114 \\
\hline
\end{tabular}




\begin{tabular}{|c|c|c|}
\hline Y-27632 & AdooQ Bioscience & A11001 \\
\hline PR-171 (Carfilzomib) & AdooQ Bioscience & A11278 \\
\hline BMS-354825 (Dasatinib) & AdooQ Bioscience & A10290 \\
\hline EdU & Thermo Fisher Scientific & A10044 \\
\hline Sulfo-Cy5-N 3 & Lumiprobe & A333 \\
\hline Buprenophrine & Provided by animal facility & $\mathrm{N} / \mathrm{A}$ \\
\hline \multicolumn{3}{|l|}{ Hydrogel reagents } \\
\hline Sodium hyaluronate & Lifecore & HA-200K \\
\hline 5-Methylfurfurylamine & TCI Chemicals & MFCD00143471 \\
\hline Methylcellulose & Spectrum Chemicals & ME137 \\
\hline Vitronectin peptide & Synthesized in house & $\mathrm{N} / \mathrm{A}$ \\
\hline MMP-degradable cross-linker & Synthesized in house & $\mathrm{N} / \mathrm{A}$ \\
\hline Silica beads & VWE & CA75873-698 \\
\hline Hyaluronidase & Sigma-Aldrich & H3884 \\
\hline \multicolumn{3}{|l|}{ Cell culture reagents } \\
\hline Essential 8 & Prepared in house & $\mathrm{N} / \mathrm{A}$ \\
\hline Matrigel & Corning & 354230 \\
\hline Dispase & Stem Cell Technologies & 7913 \\
\hline Medium 231 & Thermo Fisher Scientific & $M-231-500$ \\
\hline Smooth Muscle Growth Supplement & Thermo Fisher Scientific & S00725 \\
\hline Trypsin & Thermo Fisher Scientific & 25300062 \\
\hline Collagenase from Clostridium histolyticum & Sigma-Aldrich & $\mathrm{C} 0130$ \\
\hline $\begin{array}{l}\text { Ethyl 3-aminobenzoate methanesulfonate } \\
\text { (Tricaine) }\end{array}$ & Sigma-Aldrich & E10521 \\
\hline Accutase & Stem Cell Technologies & 7920 \\
\hline \multicolumn{3}{|l|}{ Commercial kits and materials } \\
\hline VEGF-D DuoSet ELISA Kit & R\&D Systems & DY622 \\
\hline BOLT 4-12\% $1 \mathrm{~mm}, 10$-well gel & Thermo Fisher Scientific & NW04120BOX \\
\hline Stain-Free 4-20\% 1mm 15-well gel & Bio-Rad & 4568096 \\
\hline NucleoSpin® RNA 740955.250 D-Mark Bio & Machery-Nagel & 740955.250 \\
\hline \multicolumn{3}{|l|}{ Cell models } \\
\hline H9 human embryonic stem cell, WT & WiCell & WB67614 \\
\hline H9 TSC2- & Generated in house (14) & $\mathrm{N} / \mathrm{A}$ \\
\hline H9 teratoma-derived LAM cells (WT and TSC2 ${ }^{-1-}$ ) & Generated in house & $\mathrm{N} / \mathrm{A}$ \\
\hline \multicolumn{3}{|l|}{ Animal models } \\
\hline 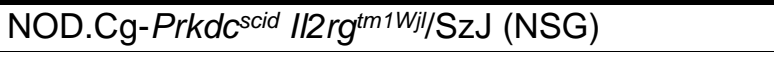 & Jackson Laboratory & 005557 \\
\hline casper mutant zebrafish & $\begin{array}{l}\text { Gift from Dr. Leonard Zon } \\
\text { (41) }\end{array}$ & $\mathrm{N} / \mathrm{A}$ \\
\hline \multicolumn{3}{|l|}{ Software and algorithms } \\
\hline ImageJ $1.53 \mathrm{c}$ & ImageJ & $\mathrm{N} / \mathrm{A}$ \\
\hline R 4.0.3 & $\mathrm{R}$ & $\mathrm{N} / \mathrm{A}$ \\
\hline RStudio 1.3.1093 & RStudio & $\mathrm{N} / \mathrm{A}$ \\
\hline Gene Set Enrichment Analysis (GSEA) & (43) & $\mathrm{N} / \mathrm{A}$ \\
\hline Mechanism of Action Miner (Elion ${ }^{\mathrm{TM}}$ ) & BioSymetrics & Described here \\
\hline
\end{tabular}

945 Cell culture

946 Pluripotent stem cell culture 
$\mathrm{H} 9 \mathrm{hPSCs}$ (female) were maintained on a thin layer of $0.16 \mathrm{mg} / \mathrm{mL}$ Matrigel at $37^{\circ} \mathrm{C}$,

$94810 \% \mathrm{CO}_{2}$. Cells were fed with Essential 8 media, prepared in house. Cells were passaged by

949 incubation with $500 \mu \mathrm{M}$ EDTA for $3 \mathrm{~min}$., then cell scraping and transfer to a new pre-coated

950 plate by wide-bore pipette.

951

$952 \quad$ LAM and control cell model derivation and culture

953 LAM cell models were established via a previously reported in vivo differentiation

954 protocol of human pluripotent stem cells (23). We differentiated a previously reported pair of

955 mCherry $^{+}$WT and genome-engineered $T S C 2^{-/-}$hPSCs, derived from the H9 parental lineage

956 (female cells) (14). First, we generated teratomas in female NOD.Cg-Prkdc ${ }^{\text {scid }} I l 2 \mathrm{rg}^{\text {tml } \mathrm{Wjl} / \mathrm{SzJ}}$

957 (NSG) mice as described in Mouse teratoma formation section. At end point, mice were

958 euthanized and dissected under sterile conditions. The teratomas were extracted while carefully

959 ensuring minimal mouse tissue remnants. The teratoma was minced and then rotated in a $5 \mathrm{U} / \mathrm{mL}$

960 Dispase solution at $37^{\circ} \mathrm{C}$ for 30 mins. Digested tissue was plated on a thin layer of $0.16 \mathrm{mg} / \mathrm{mL}$

961 Matrigel at $37^{\circ} \mathrm{C}, 5 \% \mathrm{CO}_{2}$ in Medium 231 containing Smooth Muscle Growth Supplement.

962 Tissue clumps were removed the following day. The remaining monolayer was expanded and

963 passaged by treatment with $0.05 \%$ Trypsin for $5 \mathrm{~min}$. Maintenance culture conditions included a

964 thin layer of $0.16 \mathrm{mg} / \mathrm{mL}$ Matrigel at $37^{\circ} \mathrm{C}, 5 \% \mathrm{CO}_{2}$ in Medium 231 containing Smooth Muscle

965 Growth Supplement. Cells were expanded for two passages before cryopreservation and use in

966 subsequent experiments at passages 3-5.

967 
LAM cells were clonally isolated by limiting dilution. Briefly, bulk cell cultures were

970 dissociated and serially diluted to a concentration of $\sim 0.3$ cells / $100 \mu \mathrm{L}$. We used this

971 concentration to optimize number of single cells isolated while minimizing two or more cells

972 contributing to a single clone. We added $100 \mu \mathrm{L}$ of the suspension to each well of a 96-well plate

973 containing a thin layer of $0.16 \mathrm{mg} / \mathrm{mL}$ Matrigel. Clones were expanded for 10 days before

974 dissociating and plating onto the hydrogel.

975

976 Hydrogel culture

977 Reagent production

978 Hydrogel culture was conducted according to a previously established protocol (18).

979 Briefly, a hyaluronic acid polymer backbone was derivatized with 5-methylfurfurylamine to $65 \%$

980 substitution (confirmed by ${ }^{1} \mathrm{H}$ NMR). A vitronectin-mimetic peptide (maleimide)-

981 KGGPQVTRGDVFTMPG, and MMP-degradable peptide crosslinker (maleimide)-

982 KKGRGPQGIWGQKGPQGIWGQ-K(maleimide)S were synthesized using microwave-assisted

983 Fmoc solid phase peptide synthesis with a CEM Liberty Blue automated peptide synthesizer.

984 Hydrogel viscoelasticity was increased by incorporation of methylcellulose derivatized with

985 reactive thiol groups.

986

987

Hydrogel gelation and culture

All chemically synthesized hydrogel components were mixed to the following final

989 concentrations: $0.9 \%$ methylfuronated hyaluronate, $2.3 \mathrm{mM}$ MMP crosslinker, $100 \mu \mathrm{M}$

990 vitronectin peptide, and $0.05 \mathrm{mg} / \mathrm{mL}$ thiolated methylcellulose. $15 \mu \mathrm{L}$ of the solution was added

991 to each well of a 384-well plate and permitted to gel at $37^{\circ} \mathrm{C}$ for 3 hours. Following gelation, 
992 wells were hydrated with PBS and then subjected to three media washes interspaced with

993 incubations at $37^{\circ} \mathrm{C}$ for 45 mins. LAM or control cells were then dissociated, added to plates

994 containing hydrogel, and spun for $3 \mathrm{~min}$. at $10 \mathrm{~g}$ to achieve immediate contact with the hydrogel.

995

996 Cell treatments

997 Drug treatments

All small molecule compounds were diluted in either DMSO or PBS, unless otherwise

999 stated. The appropriate diluent-matched vehicle control was included in every experiment. Drug

1000 treatments were added directly to wells containing cells at $5 \mathrm{X}$ concentrations to avoid washing

1001 off any cells, particularly in sensitive miniaturized formats. Rapamycin was consistently used at

1002 a 20nM concentration. All compound treatments were conducted for 72 hours unless otherwise

1003 stated.

1004

1005 Live cell staining

1006 Live cell staining dyes were used at the following final concentrations: $10 \mu \mathrm{g} / \mathrm{mL}$

1007 Hoechst 33342, 50 nM SyTOX Green, $4 \mu$ M Caspase-3 Enzyme Substrate, and 0.2 $\mu \mathrm{g} / \mathrm{mL}$

1008 Annexin V. Dyes were incubated for $30 \mathrm{~min}$. prior to imaging and added as 10X concentrates in

1009 PBS; Annexin V diluent also contained $2.5 \mathrm{mM} \mathrm{CaCl}_{2}$. To avoid cell detachment in the

1010 miniaturized well format, live imaging dyes were not washed prior to imaging; this did not

1011 impact image acquisition as dyes are minimally fluorescent unless bound to the target molecule.

1012

1013 Cytotoxicity-invasion assay 
1015 incubated at $37^{\circ} \mathrm{C}$ and $5 \% \mathrm{CO}_{2}$. At end point, Hoechst and SyTOX were added directly to all

1016 wells as described in Cell treatments section. Whole-well multi-planar images were acquired by

1017 widefield microscopy with $40 \mu \mathrm{m}$ separation between z-stacks. Following image acquisition,

1018 wells were fixed overnight in $10 \%$ formalin. We then added $1 \mu \mathrm{g}$ of silica beads to each well and

1019 acquired multiplanar brightfield images, with the plane of maximal contrast used to determine

1020 hydrogel-liquid interface (described in Image analysis). Acquiring location of the hydrogel

1021 interface (i.e., start of the cellular position) is essential for accurate invasion distance calculation;

1022 the hydrogel exhibits a meniscus which leads to a variable $\mathrm{Z}$ starting position depending on the

1023 XY location.

1024

1025 Three-dimensional drug screen

1026 Screen design

1027 Both WT and $T S C 2^{-/-}$cells were treated with every drug from the Ontario Institute for

1028 Cancer Research (OICR) Kinase Inhibitor and Tool Compound library (total of 800 compounds)

1029 at a concentration of $5 \mu \mathrm{M} \pm 20 \mathrm{nM}$ rapamycin. Cells were treated for $72 \mathrm{hr}$. while cultured in

1030 hydrogel and assessed at end point for cytotoxicity and invasion modulation as described in

1031 Cytotoxicity-invasion assay. Each plate included internal vehicle-treated only controls. Z' was

1032 calculated for cytotoxicity and invasion modulation using vehicle-treated samples (negative

1033 control), $10 \mu \mathrm{M}$ Y27632-treated (positive control, invasion), and $5 \mu \mathrm{M}$ Carflizomib-treated

1034 (positive control, cytotoxicity).

1035

1036 Compound score calculation 
To identify drugs with statistically significant effect(s), we computed z-scores for

1038 invasion modulation, cytotoxicity, selective invasion modulation, and selective cytotoxicity, for

1039 each WT and $T S C 2^{-/}$in the presence or absence of rapamycin. We confirmed that the reference

1040 population of vehicle-treated controls for each metric was normally distributed and variance did

1041 not vary with effect mean. Cytotoxicity is determined by the percentage of SyTOX ${ }^{+}$cells;

1042 invasion modulation is determined by the percentage of cells invading past the vehicle-control

1043 median threshold (See Image analysis section). Selective cytotoxicity is determined by the

1044 difference in cytotoxicity between WT and $\mathrm{TSC}^{-/}$cells, where positive values indicate more

1045 dead cells in the TSC $^{-\%}$ condition. Selective invasion modulation is determined by the difference

1046 in cytotoxicity between $T S C 2^{-/-}$and WT cells, where positive values indicate fewer invading

1047 cells in the $T S C 2^{-/}$condition. We then calculated p-values and corrected for multiple hypothesis

1048 testing by computing false discovery rates. All computation was performed using R 4.0.3 and

1049 RStudio 1.3.1093.

1050

1051 Target enrichment analysis

1052 To refine our candidate compound list, we performed target enrichment analysis using a

1053 modified version of the GSEA algorithm (43). Enrichment analysis was performed separately for

1054 cytotoxicity and invasion modulation. For cytotoxicity, we focused our compound list to drugs

1055 that showed selective cytotoxicity, either in the presence or absence of rapamycin. If a drug was

1056 shown to be significantly beneficial in one condition but significantly detrimental in the other, it

1057 was excluded. We then derived a singular compound score by computing the arithmetic mean

1058 across the two conditions. Similarly, for invasion modulation, we focused our compound list to

1059 drugs that exhibit anti-invasion effects towards WT or $T S C 2^{-/}$cells, either in the presence or 
1060 absence of rapamycin. Again, we excluded compounds that showed opposing effects, and

1061 derived a singular compound score by arithmetic mean across conditions.

1062

We next generated a background target list using known compound targets as annotated

1063 by the OICR. We created generalizable categories wherever possible, however, there were many

1064 targets that could not be grouped and conferred an $n=1$ category. To avoid the possibility of

1065 bias, these categories were established by an independent author blinded to the original

1066 compound results. Using this background list and our compound score lists as described above,

1067 we determined target enrichment using the GSEA algorithm (43).

1068

1069

Elion $^{T M}$ analysis

1070 A limitation to our analyses is the small number of compounds which were identified to

1071 selectively eliminate $T S C 2^{-/}$cells. We sought to extend our compound list in silico using a

1072 structure-based approach with Elion ${ }^{\mathrm{TM}}$ (Mechanism of Action Miner), conducted by an

1073 independent group. Elion ${ }^{\mathrm{TM}}$ is a software package that ingests binary phenotypic data linked to

1074 individual drug treatments to suggest possible underlying protein targets and molecular

1075 pathways. The platform inputs phenotypic screening data in the form of a two-column CSV file

1076 corresponding to the chemical structure in SMILES format alongside a binary bioactivity

1077 reading.

1078

Using this dataset, a total of 8,000 features are generated for each supplied chemical

1079 structure. These features are comprised of chemical fingerprints (ECFP4, FCFP4, RDK-layered

1080 fingerprint, and MACCS) alongside physical properties (e.g., molecular weight, total polar

1081 surface area, $\log$ P). If there are fewer than 8,000 rows in the input data set, a subset of features

1082 are chosen for downstream machine learning. The size of this feature subset is set to be $70 \%$ of 
1083 the number of rows in the input data set. Feature selection is performed using a bootstrapped

1084 logistic regression strategy. In brief, a set number of features are sampled from the original

1085 feature set and are used to train a logistic regression model. The coefficients of this model are

1086 then used to rank feature importance. This process is repeated 5,000 times and the resulting

1087 coefficients are averaged for each feature to create a summarized feature importance score.

1088 Once a feature set is chosen, a total of 6 machine learning models are built and evaluated

1089 on the input data set (XGBoost, random forest, Gaussian naive Bayes, uniform and distance

1090 weighted K-nearest neighbours, and Gaussian process classifiers). Each model is trained and

1091 evaluated using 10-fold cross validation while recording classification performance according to

1092 accuracy, ROC-AUC, precision and recall. The best performing model is then chosen and used

1093 to rank a set of 1 million compounds (curated from public databases) according to probability of

1094 inducing the given phenotype.

1095 Of the $1 \mathrm{M}$ ranked compounds, several are annotated according to experimentally

1096 validated protein targets and mechanisms of action. The GSEA algorithm is used to determine

1097 which of these targets and MoAs are most positively enriched within the ranked set of

1098 compounds. We then subset this list using an FDR threshold to identify a set of enriched targets

1099 and MoAs. Using the enriched targets, we perform gene ontology and protein family pathway

1100 enrichment using the Fisher exact test. A Bonferroni corrected p-value threshold of 0.05 is used

1101 to identify cellular pathways corresponding to the phenotype of interest.

1102 As a result of this process, Elion ${ }^{\mathrm{TM}}$ translates an input phenotypic screen into three

1103 informative outputs. First, it supplies a ranked list of publicly available compounds prioritized

1104 according to their likelihood to induce the given phenotype. Second, it provides a list of targets

1105 and MoAs likely to mitigate the provided phenotype. Last, it annotates these targets with 
1106 enriched cellular pathways. All of these results are presented in a web application annotated with

1107 rich descriptions and link-outs to relevant genetic databases.

1109 Image analysis

1110 Identification of cell spatial positions and cell invasion distance

1111 We identified XYZ cell positions in the hydrogel by analysis of the Hoechst channel z-

1112 stack. We first determined XY positions by employing the ImageJ 1.53c "Find maxima" function

1113 on the z-stack maximum intensity projection. We automated the determination of the noise (or

1114 background) threshold by empirical iteration. Using the assumption that true Hoechst signal

1115 should be substantially above background fluorescence, we computed "Find maxima" with a

1116 liberal threshold, and then progressively increased threshold stringency until the number of

1117 identified points did not vary with each stepwise threshold change. Each maxima was determined

1118 to correspond to a single cell spatial location. Following, for each XY spatial position, we

1119 iterated through the Hoechst z-stack and identified to the point of maximal intensity,

1120 corresponding to the cell $\mathrm{Z}$ position.

1121 To determine the invasion distance of each single cell, we must first know the cell

1122 starting position, which varied across XY positions due to the meniscus exhibited by the

1123 hydrogel. To identify determine hydrogel interface $\mathrm{Z}$ position across the XY plane, we used the

1124 silica bead brightfield images (described in Cytotoxicity-invasion assay). For each XY cell

1125 spatial position, we iterated through the brightfield z-stack and identified the point of maximal

1126 contrast, which corresponded to the layer containing silica beads (due to diffraction). We then

1127 determined individual cell distances travelled by computing the difference between cell starting 
1128 and final positions. This process, automated for high throughput analysis, was scripted in ImageJ

$11291.53 \mathrm{c}$.

1131 Binarization of live cell stains

1132 Live cell stains (i.e. SyTOX, Caspase-3 enzyme substrate, and Annexin V) were

1133 binarized into a positive or negative signal for each cell. We first created a masking around the

1134 Hoechst signal of each cell in the maximum intensity projection image, then measured the total

1135 fluorescent signal of the live cell stain within each masking. To binarize in an automated fashion,

1136 we fit an empirical probability density function (ePDF) by kernel density estimation on the

1137 vehicle control sample values. Assuming the majority of untreated samples should be negative

1138 for cell death stains, we determined the threshold for binarization to by the first local minimum

1139 of the negative control ePDF. Cells across conditions were then binarized according to their

1140 matched control threshold. This process, automated for high throughput analysis, was scripted in

1141 Image J 1.53c and R 4.0.3 within the RStudio 1.3.1093 environment.

1143 Quantification of cellular invasion

1144 Cellular invasion was determined by number of cells invading past a fixed distance. As

1145 the invasion distance varied slightly batch to batch (see Fig. 1F), distance thresholds for each

1146 experiment were based on within-experiment vehicle controls. We used both the median

1147 invasion of vehicle controls, which is sensitive to detecting decreases in invasion, and $90^{\text {th }}$

1148 percentile invasion of vehicle controls, which is sensitive to detecting increases in invasion.

1149 Genotype-specific thresholds were employed due to the differing invasion distances between WT 1150 and $T S C 2^{-/}$cells. To determine invasion of alive cells only, SyTOX ${ }^{+}$positive cells were removed 
1151 from the distribution prior to calculation of invasion percentages. This process, automated for

1152 high throughput analysis, was scripted in R 4.0.3 within the RStudio 1.3.1093 environment.

1154 Immunofluorescence stain quantification

1155 Immunofluorescence experiments were quantified using the raw image files, ensuring the

1156 absence of detector saturation. We created a masking around the Hoechst signal of each cell in

1157 the maximum intensity projection image, then measured the mean fluorescent signal of the

1158 protein-of-interest within the total masking area. Measurements per replicate were re-scaled from

$11590-1$ by dividing by the replicate maximum value. We note that comparisons between plastic

1160 and hydrogel samples cannot be directly made, as the imaging parameters differ between the

1161 two-dimensional vs. three-dimensional environment (see Fig. 2B).

\section{Invasion quantification upon zebrafish xenotransplantation}

1164 Invasion of transplanted $\mathrm{mCherry}^{+}$cells was performed in a semi-automated fashion on

1165 blinded images (Fig. S6B). For each image, a region of interest was manually selected on the

1166 maximum intensity $\mathrm{Z}$ projection, to distinguish areas with $\mathrm{mCherry}^{+}$cells from surrounding

1167 auto-fluorescent regions (e.g., zebrafish eye, yolk sac, ossicle). Images were then binarized using

1168 a constant threshold to distinguish positive signal from background. Pixels were classified into

1169 "invaded" or "not invaded" based on the distance from the center of the initial injection site. We

1170 used the average of the first local minima of the positive pixel histogram from 1 day post

1171 injected images to determine the distance for classification of invaded or not. Following pixel

1172 classification, the ratio of the total positive pixel intensity in each group was computed to 
1173 determine the zebrafish invasion score. Groups were then unblinded and graphed. This process

1174 was scripted in Image $1.53 \mathrm{c}$ and R 4.0.3 within the RStudio 1.3.1093 environment.

1176 RNA-seq

1177 RNA extraction and quality control

1178 Extraction of RNA from cells embedded in hydrogel is made challenging by the low

1179 cellular density relative to the abundant extracellular matrix. To extract RNA, we developed an

1180 extraction protocol that combines phenol-chloroform phase separation with column-based

1181 purification. We first added TriZOL directly to wells and homogenized the cell-hydrogel mixture

1182 using a 26-gauge needle. We then centrifuged the lysate for $5 \mathrm{~min}, 12,000 \mathrm{~g}$, at $4^{\circ} \mathrm{C}$ to pellet the

1183 cross-linked hyaluronic acid matrix. We extracted the supernatant and mixed in chloroform,

1184 followed by centrifugation to induce phase separation. The colourless aqueous phase was

1185 extracted and mixed with equal volumes of $70 \% \mathrm{EtOH}$. Following a brief incubation at room

1186 temperature, the solution was eluted through a Machery-Nagel Nucleospin column. We

1187 proceeded with column-based purification as per manufacturing protocol.

RNA-sequencing and raw data processing

1190 RNA samples were shipped to the Donnelly Sequence Centre (Toronto, Canada) for

1191 RNA quality-control, library preparation, and next-generation sequencing. RNA integrity was

1192 assessed via Bioanalyzer (Agilent) and only samples with RIN > 8 were prepared for sequencing.

1193 Oligo(dT) priming via SMART-Seq v4 (Takara Bio) preparation kit was used to generate full-

1194 length cDNA libraries. Samples were subjected to paired-end sequencing on a NovaSeq 6000,

1195 100c (Illumina) to a depth of $\sim 50$ million reads per sample. 
Raw sequence read quality was assessed by FastQC. Read feature assignments and duplication rates were determined using featureCounts and Picard. Overall mapping rate was

1198 assessed with HISAT2. Finally, read assignment to transcripts was performed using Salmon,

1199 generating a final pseudocount abundance matrix. All QC processing was summarized using

1200 MultiQC and programmed in R 4.0.3 within the RStudio 1.3.1093 environment. RNA-seq data is 1201 accessible at the Gene Expression Omnibus (GEO) repository with accession GSE179044.

\section{Differential gene expression and enrichment analysis}

1204 Pseudocount abundance data generated by Salmon was imported into the DEseq2

1205 framework in R for differential expression and enrichment analysis. Principal components

1206 analysis was conducted on all samples to visualize transcriptomes in a two-dimensional space.

1207 For single variable differential expression testing, we subsetted samples to only include the

1208 untreated and fit the following model: batch + genotype + substrate. We then tested for genes

1209 with significant coefficients by Wald test, separately for genotype and for culture substrate. To

1210 assess for changes across genotype that differs between matrix condition, we again subsetted for

1211 untreated samples and fit the following model: $\sim$ batch + genotype + substrate +

1212 genotype:substrate. The interaction term coefficient for each gene was tested for significance by

1213 Wald test. Differentially expressed genes were called when false discovery rate (FDR) $<0.05, \pm$ $1214 \mid \log 2$ FoldChange $\mid>1$ (as indicated in the text).

1215 To visualize expression values by heatmap or gene cluster, sample conditions were

1216 collapsed by abundance summation, normalized, and then transformed by regularized $\log _{2}$

1217 transformation (implemented in DEseq2). Heatmaps were generated using the pheatmap package

1218 in R and gene clusters were generated by hierarchal clustering. GO term enrichment was 
1219 performed using clusterProfiler on significant DEGs (FDR $<0.05, \pm \mid \log 2$ FoldChange $\mid>1)$. All

1220 analysis was conducted using R 4.0.3 within the RStudio 1.3.1093 environment.

\section{Animal studies}

1223 Mouse teratoma formation

1224 hPSCs were dissociated into single cells by Accutase treatment for $15 \mathrm{~min}$ at $37^{\circ} \mathrm{C}$.

1225 Single cells were harvested, washed, and resuspended in $5 \mathrm{mg} / \mathrm{mL}$ Matrigel. Female 8-week-old

1226 NSG mice were treated with buprenophrine 1 hour before injection, then anesthetized by

1227 isofluorane under a continuous stream of $\mathrm{O}_{2}$. We bilaterally injected $1 \times 10^{6}$ hPSC into the mouse

1228 tibialis anterior. We allowed teratomas to grow over a 12-week period, after which mice were

1229 sacrificed and teratomas extracted.

$1231 \quad$ Mouse subcutaneous xenografts

1232 LAM cells were dissociated into single cells by $0.05 \%$ Trypsin treatment for $5 \mathrm{~min}$. at

$123337^{\circ} \mathrm{C}$, washed, and resuspended in $5 \mathrm{mg} / \mathrm{mL}$ Matrigel. Female 8-week-old NSG mice were

1234 anesthetized by isofluorane under a continuous stream of $\mathrm{O}_{2}$ and injected with $1 \times 10^{6}$ cells

1235 subcutaneously in each rear flank. We monitored for palpable tumor growth weekly over a four-

1236 month period, after which animals were sacrificed.

1238 Mouse IVIS image acquisition

1239 Female 8-week-old NSG mice that were injected with LAM cells in each rear flank were

1240 monitored for tumor growth by endogenous mCherry expression of LAM cells. Mice were

1241 anesthetized by isofluorane under a continuous stream of $\mathrm{O}_{2}$ and shaved to eliminate background 
1242 fluorescence from the fur coat. Mice were then imaged at fixed exposure times by in vivo

1243 imaging (PerkinElmer, IVIS®).

1245 Zebrafish toxicity assay

1246 72-hour post-fertilization (hpf) zebrafish larvae were arrayed one larva per well in a 96-

1247 well plate and treated with increasing concentrations of each inhibitor for 72 hrs to ascertain

1248 toxicity thresholds. There were no in vivo toxic effects at the experimental in vitro concentrations

1249 and thus, zebrafish experimental doses were chosen to stay consistent with in vitro treatment

1250 doses.

1251

1252 Zebrafish hindbrain ventricle xenotransplantation

1253 For each injection experiment, a separate cryovial of cells was thawed and cultured 3

1254 days prior to zebrafish transplantation, without any subculturing. On the day of transplantation,

1255 cells were dissociated by $0.25 \%$ trypsin, centrifuged for 5 mins at $300 g$, and resuspended in

1256 approximately $30 \mu \mathrm{L}$ of culture medium for injection. $72 \mathrm{hpf}$ zebrafish larvae were anesthetized

1257 with $0.09 \mathrm{mg} / \mathrm{mL}$ tricaine (Millipore Sigma) and arrayed in troughs of an agarose injection plate

1258 and used for cell transplantation using protocols described previously $(44,45)$. The cells were

1259 backloaded into a pulled capillary needle and allowed to settle for approximately 20 mins at

$126035^{\circ} \mathrm{C}$ to ensure a cell pellet at the bottom of the needle. A PLI-100A Pico-liter Microinjector

1261 (Warner Instruments) was used to manually inject 50-100 cells into the hindbrain ventricle

1262 (HBV) of each larva. Following injections, the larvae were kept at $35^{\circ} \mathrm{C}$ for the remainder of the

1263 experiment. 
1 day post injection (dpi), injected larvae were screened on an Axio Observer 7

1267 fluorescent microscope under an mCherry filter to ensure the presence of cells only in the HBV.

1268 Groups of 20-30 positively injected larvae were randomized into groups to be treated with either

1269 vehicle control (DMSO), $20 \mathrm{nM}$ rapamycin alone, $5 \mu \mathrm{M}$ SB939 alone, $20 \mu \mathrm{M}$ SAHA alone, 1

$1270 \mu \mathrm{M}$ LBH589 alone or with one HDACi in combination with rapamycin by immersion therapy

1271 for $72 \mathrm{hrs}$. At the experimental endpoint (3 days post-treatment) the groups of larvae were

1272 blinded and imaged on the Axio Observer 7 using the z-stack function to capture cell movement

1273 in all planes. Blinded groups of images were then subjected to automated invasion analysis by an

1274 independent study author.

1275

Zebrafish whole larval dissociation and fixation

1277 At $1 \mathrm{dpi}$ (baseline) and $4 \mathrm{dpi}$ (three days post-treatment), 20 larvae from each group were 1278 euthanized and dissociated in $100 \mathrm{mg} / \mathrm{mL}$ collagenase solution for approximately 30 mins. Upon

1279 completion of dissociation (i.e., single cell suspension formed), $200 \mu \mathrm{L}$ of $100 \%$ FBS was added

1280 to slow the enzymatic reaction. The samples were then centrifuged for $5 \mathrm{~min}$. at $300 \mathrm{~g}$ and the

1281 supernatant was removed, leaving a pellet of human tumor cells among the zebrafish cells. The

1282 samples were washed once in $30 \%$ FBS in PBS and centrifuged once more for $5 \mathrm{~min}$. at $300 \mathrm{~g}$.

1283 The supernatant was removed and $250 \mu \mathrm{L}$ of $4 \%$ PFA in PBS was added to each sample for 20

1284 min. in the dark. $1 \mathrm{~mL}$ of PBS was added and samples were centrifuged and PFA supernatant was

1285 removed. Samples were resuspended in $500 \mu \mathrm{L}$ PBS, stored at $4^{\circ} \mathrm{C}$, and blinded prior to flow

1286 cytometry analysis. 


\section{Immunofluorescence staining}

The following protocol is for immunofluorescence staining of cells in monolayer culture

1290 on plastic. Modifications for whole-mount (WM) staining of cells in three-dimensional hydrogel

1291 are indicated throughout.

Cells were fixed with 4\% PFA for 15 min. (WM: 30 min.) at room temperature. Wells

1293 were washed 3 x 5 min. (WM: 20 min.) with PBS, then permeabilized with $0.1 \%$ Triton-X in

1294 PBS for $20 \mathrm{~min}$. (WM: $40 \mathrm{~min}$ ) at room temperature. Wells were washed 3 x 5 min. (WM: 20

1295 min.) with PBS, then blocked with 1\% BSA in PBS for $1 \mathrm{hr}$. (WM: $2 \mathrm{hr}$.) at room temperature.

1296 We then added primary antibody diluted in blocking solution for overnight incubation at $4{ }^{\circ} \mathrm{C}$.

1297 The following concentrations of antibodies were employed: PMEL (1:50), ACAT2 (1:100),

$1298 \operatorname{pS} 6 R P^{\operatorname{Ser} 235 / 236}(1: 100)$, and p4E-BP1 ${ }^{\text {Thr37/46 }}(1: 200)$. The next day, wells were wash $3 \times 5$ min.

1299 (WM: 5 x 30 min.) with PBS, then incubated with fluorescent secondary antibodies diluted

1300 blocking solution for $1 \mathrm{hr}$. (WM: $2 \mathrm{hr}$.) at room temperature. All fluorescent secondary

1301 antibodies were used at a 1:1000 dilution. Wells were then washed 3 x 5 min. (WM: 5 x 30 min.)

1302 with PBS then counterstained with $10 \mu \mathrm{g} / \mathrm{mL}$ Hoechst 33342 for $30 \mathrm{~min}$. (WM: 45 min.) Wells

1303 were washed 3 x 5 min (WM: 5 x 30 min.), then mounted with a 90\% glycerol (WM: PBS, as the

1304 hydrogel disintegrates in glycerol) solution made in house, prior to imaging.

1305

1306 Enzyme-linked immunosorbent assay (ELISA)

1307 Maintenance cultures of cells at equivalent densities were incubated for $16 \mathrm{hr}$. in Medium

$1308231 \pm 20$ nM rapamycin, without serum supplement. Conditioned media was collected and

1309 centrifuged to remove any cellular debris, then assayed by VEGF-D ELISA kit (R\&D Systems,

1310 DY622) following the manufacturer protocol. 
1312 Flow cytometry

$131437^{\circ} \mathrm{C}$, washed, and then fixed with $4 \%$ PFA for $15 \mathrm{~min}$. at room temperature. Fixing solution was

1315 diluted out 1/10 in PBS, cells were pelleted by centrifugation, and supernatant discarded. For

1316 details on zebrafish single cell preparation, see Zebrafish whole organism dissociation and

1317 fixation section. Fixed single cell suspensions were permeabilized with $0.1 \%$ Triton-X in PBS

1318 for 20 min. at room temperature. Permeabilizing solution was diluted out 1/10 in PBS, cells were

1319 pelleted by centrifugation, and supernatant discarded. Samples were then blocked with $1 \%$ BSA

1320 or 5\% Goat Serum in PBS for $1 \mathrm{hr}$. Cells were pelleted by centrifugation, supernatant discarded,

1321 and primary antibodies diluted in blocking solution were added for overnight incubation at $4^{\circ} \mathrm{C}$.

1322 The following concentrations of antibodies were employed: PMEL (1:50), ACAT2 (1:100),

1323 mCherry (1:1000), and Cleaved CASP3 (1:500). The next day, primary antibody solution was

1324 diluted out 1/10 in PBS, cells were pelleted by centrifugation, and supernatant discarded.

1325 Samples were then incubated with fluorescent secondary antibodies diluted blocking solution for

$13261 \mathrm{hr}$. at room temperature. All fluorescent secondary antibodies were used as a 1:1000 dilution.

1327 Secondary antibody solution was diluted out 1/10 in PBS, cells were pelleted by centrifugation,

1328 and supernatant discarded. Samples were next counterstained with $10 \mu \mathrm{g} / \mathrm{mL}$ Hoechst 33342 for

$132920 \mathrm{~min}$. at room temperature. Hoechst 33342 solution was diluted out 1/10 in PBS, cells were

1330 pelleted by centrifugation, and supernatant discarded. Finally, cells were strained and analyzed

1331 using the LSRFortessa (BD) flow cytometer.

\section{Low input western blot}


Hydrogel culture must be performed in a miniaturized format to maintain the appropriate mechanics as previously reported (18). Naturally, this poses a challenge for collecting sufficient

1336 protein for standard molecular biology methods, such as a western blot. To address this

1337 challenge, we developed a method for a low input western blot that includes in-well lysis and 1338 sample preparation, followed by a gel-based method for sample normalization.

Samples were cultured on plastic or in hydrogel for $72 \mathrm{hr}$. Following, sample media was

1340 aspirated to the hydrogel interface (leaving a similar volume in plastic wells) and an equivalent

1341 volume of 2X Laemmli-RiPA buffer was added to each well. Samples were incubated for 10

1342 min. at $37^{\circ} \mathrm{C}$ and triturated up and down, careful not to disturb the hydrogel. The sample volume

1343 was then extracted and boiled for $10 \mathrm{~min}$. at $70^{\circ} \mathrm{C}$. As this extraction contains a large amount of

1344 non-cellular derived protein components (due to degradation of the hydrogel MMP-cleavable

1345 crosslinkers), standard protein quantification by colorimetric methods (e.g., BCA, and Bradford)

1346 are not reliable. Instead, we performed total protein quantification on gel-separated samples.

1347 First, an aliquot of each sample was electrophoresed on a stain-free 4-20\% $1 \mathrm{~mm} \mathrm{15-well} \mathrm{gel,}$

1348 along with a serial dilution of a sample of known concentration. The gel was then activated by

1349 UV exposure and total protein visualized by ChemiDoc Gel Imager (Bio-Rad). We then

1350 calculated individual sample concentrations by comparing against the within-gel standard curve,

1351 without including bands corresponded to the hydrogel MMP peptides.

1352 After sample extraction and quantification, we analyzed samples following standard

1353 western blotting procedures. To maximize sample input, Thermo Fisher Scientific BOLT gels

1354 were used, which contain space for up to $60 \mu \mathrm{L}$ of sample per lane. We first separated samples

1355 by SDS-PAGE using a BOLT 4-12\% $1 \mathrm{~mm}$ 10-well gel and MES running buffer. Samples were

1356 transferred onto a $0.45 \mu \mathrm{m}$ PVDF membrane overnight at $4^{\circ} \mathrm{C}$ by wet transfer with Towbin 
1357 buffer (containing $0.025 \%$ SDS and 10\% MeOH). The membrane was then blocked by 5\% BSA

1358 in PBS-T (0.1\% Tween-20) for $1 \mathrm{hr}$. at room temperature. Following, the membrane was

1359 incubated overnight at $4^{\circ} \mathrm{C}$ in primary antibodies diluted in blocking buffer at the following

1360 concentrations: $p S 6 \operatorname{RP}^{\mathrm{Ser} 235 / 236}(1: 5000), \mathrm{p} 4 \mathrm{E}-\mathrm{BP} 1^{\mathrm{Thr37/46}}(1: 1000), \mathrm{S} 6 \mathrm{RP}(1: 500), 4 \mathrm{E}-\mathrm{BP} 1$

1361 (1:1000), TSC2 (1:5000), and ACTB (1:5000). The membrane was washed 3 x 5 min. with PBS-

$1362 \mathrm{~T}$, and then incubated for $1 \mathrm{hr}$. at room temperature in fluorescent secondary antibodies diluted

1363 1:10,000 in blocking buffer. The membrane was washed 3 x 5 min. with PBS-T and then imaged

1364 using Odyssey Gel Imager (LI-COR Biosciences).

1365

EdU proliferation assay

Cells were pulsed with $5 \mu \mathrm{M}$ of EdU for $3 \mathrm{hr}$. Subsequently, cells were fixed with $4 \%$

1368 PFA for $15 \mathrm{~min}$. at room temperature. Wells were washed $3 \times 5$ min. with PBS, permeabilized

1369 with $0.1 \%$ Triton-X in PBS for $20 \mathrm{~min}$. at room temperature, then washed again 3 x $5 \mathrm{~min}$. We

1370 prepared the click reaction by mixing the following components in the described order, in PBS,

1371 to the indicated final concentrations: $4 \mathrm{mM} \mathrm{Cu}_{2} \mathrm{SO}_{4}, 5 \mu \mathrm{M}$ Sulfo-Cy5-N 3 , and $100 \mathrm{mM} \mathrm{L-}$

1372 ascorbic acid. The click reaction mix was added to wells containing cells and incubated at room

1373 temperature for $30 \mathrm{~min}$. Wells were washed 3 x 5 min. with PBS, counterstained with $10 \mu \mathrm{g} / \mathrm{mL}$

1374 Hoechst 33342 for $30 \mathrm{~min}$, washed again 3 x 5 min., and then imaged.

\section{Clonogenic assay}

1377 Cells were plated on the hydrogel and treated with HDAC inhibitors at the designated

1378 concentration for $72 \mathrm{hr}$. Following treatment, wells were washed 3 x $20 \mathrm{~min}$. with media to

1379 remove the drug from solution. The hydrogel was then solubilized by addition of $150 \mathrm{U}$

1380 hyaluronidase per $15 \mu \mathrm{L}$ hydrogel and incubated for $1 \mathrm{hr}$. at $37^{\circ} \mathrm{C}$. Following, $0.05 \%$ Trypsin 
1381 was added to the wells for $10 \mathrm{~min}$. at $37^{\circ} \mathrm{C}$ to dissociate cells. Wells were triturated and then

1382 plated on two-dimensional tissue cultures plates, in serial dilution. Cells were permitted to

1383 proliferate for 10 days, forming colonies from single cells. Following, wells were fixed with $4 \%$

1384 PFA for $15 \mathrm{~min}$. at room temperature, then washed 3 x 5 min. with PBS. Colonies were stained

1385 with $0.1 \%$ crystal violet for $1 \mathrm{hr}$. at room temperature, washed $3 \times 5$ min. with $\mathrm{ddH}_{2} \mathrm{O}$, air dried, 1386 and imaged. 


\section{SUPPLEMENTARY FIGURES}
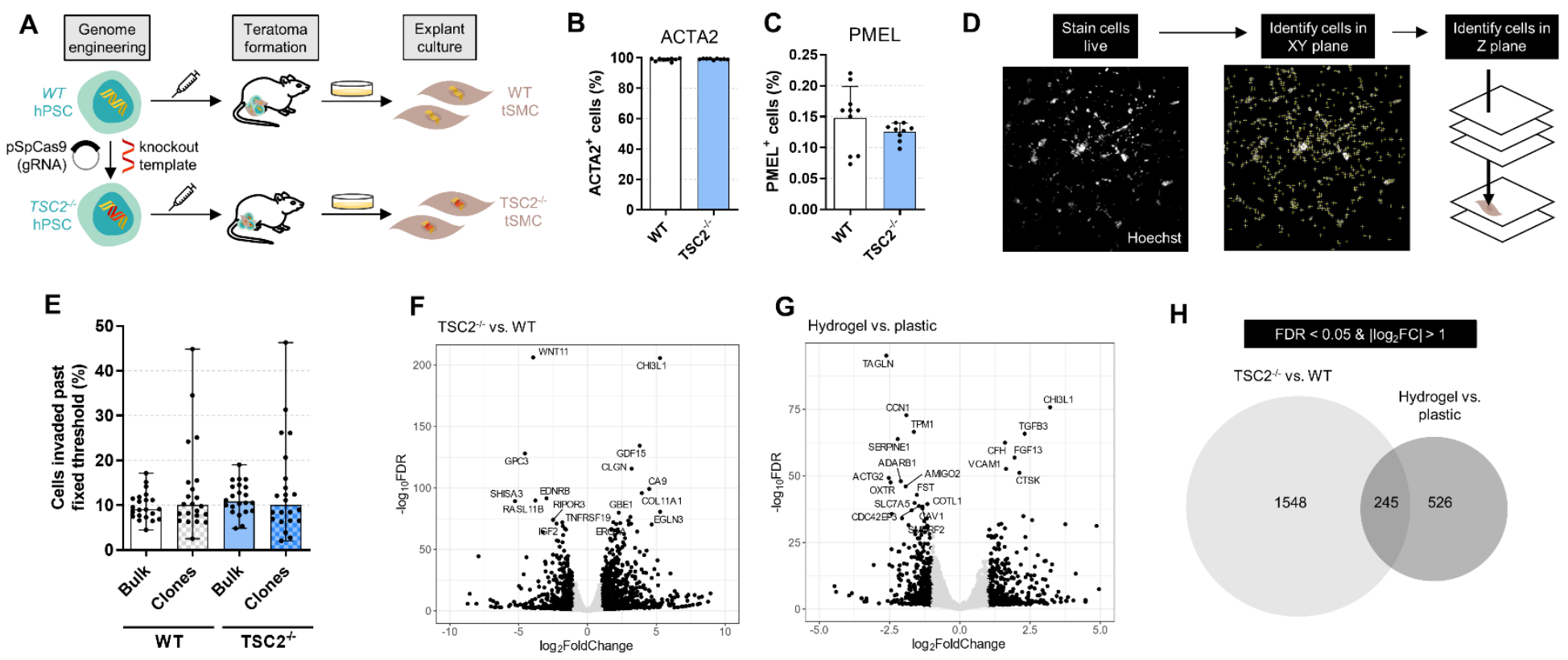

H
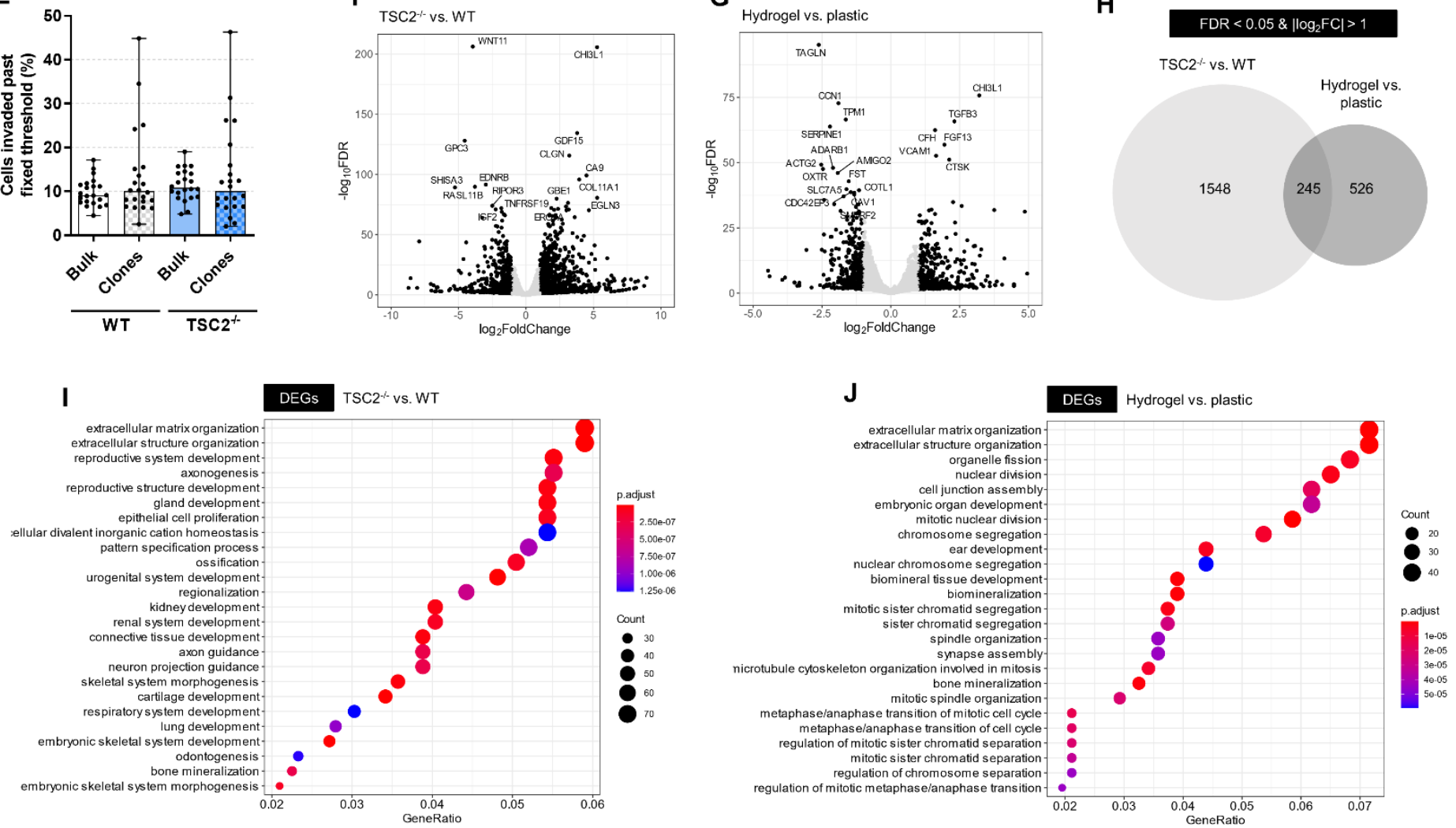

1388 Fig. S1. Hydrogel culture of stem cell-derived disease models exhibits features of LAM. (A)

1389 Schematic of generation of LAM cellular models. (B-C) Quantification of LAM markers by flow

1390 cytometry from cells in maintenance culture (mean $\pm \mathrm{SD} ; *=\mathrm{p}<0.05$ by Student $\mathrm{t}$-test; $\mathrm{n}=10$ ).

1391 (D) Schematic of cell position identification in XYZ planes. (E) Percentage of cells invaded past

1392 threshold set by $90^{\text {th }}$ percentile invasion distance of bulk cultures, following three-day hydrogel

1393 culture. Bulk cultures are maintenance cultures of LAM cell lines; clones are populations of cells

1394 expanded from a single cell isolated from maintenance cultures prior to seeding on hydrogel 
1395 (mean \pm data range; no statistical test). (F-G) Volcano plot upon comparing TSC2\% vs. WT cells

1396 (F) and hydrogel vs. plastic samples (G). Points highlighted in black are considered differentially

1397 expressed (FDR $<0.05$ and $\left.\left|\log _{2} \mathrm{FC}\right|>1\right)$. The 20 most significantly DEGs are noted. $(\mathrm{H})$

1398 Overlap in DEG between genotype and culture substrate gene lists; genes considered as DEGs if

1399 FDR $<0.05$ and $\left|\log _{2} \mathrm{FC}\right|>1$. (I-J) Dotplot of GO term enrichment analysis of DEG lists (FDR <

$1400 \quad 0.05$ and $\left.\left|\log _{2} \mathrm{FC}\right|>1\right)$ upon comparing $T S C 2^{-/-}$vs. WT cells (I) and hydrogel vs. plastic samples

1401 (J). The 25 most significantly enriched terms are plotted. 
A

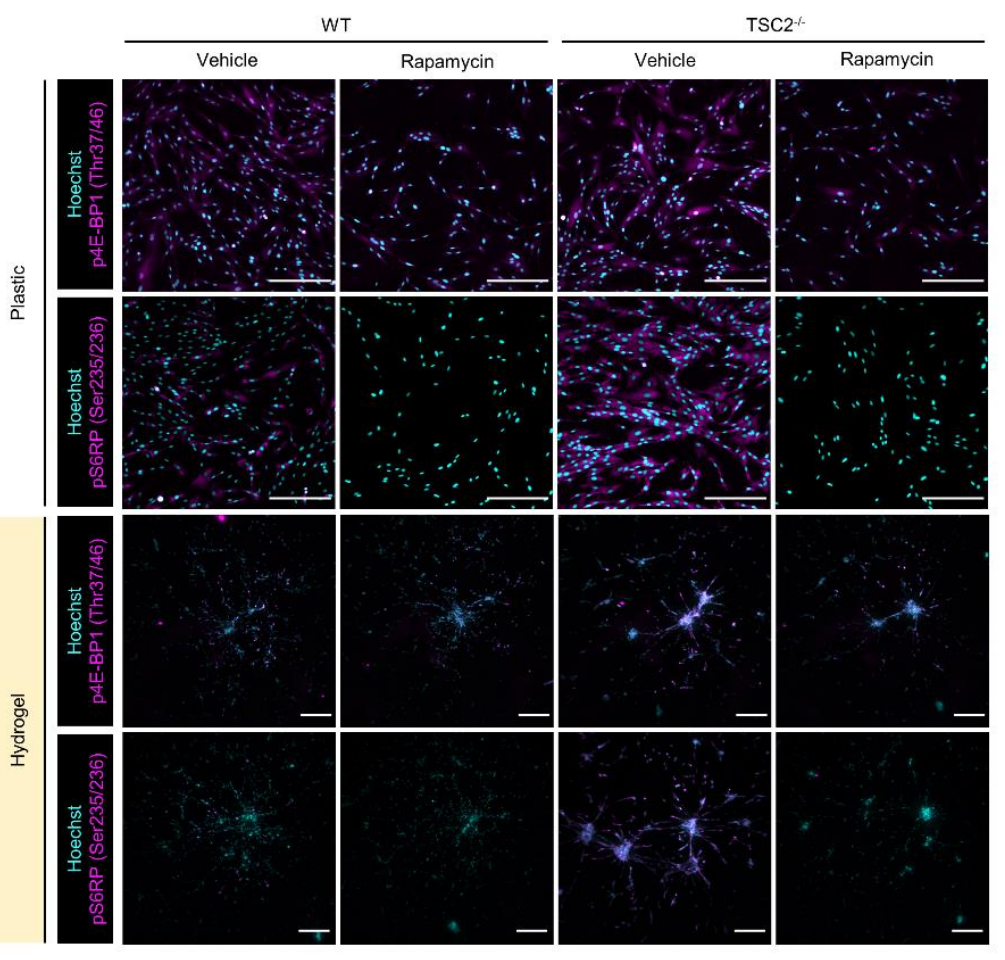

B

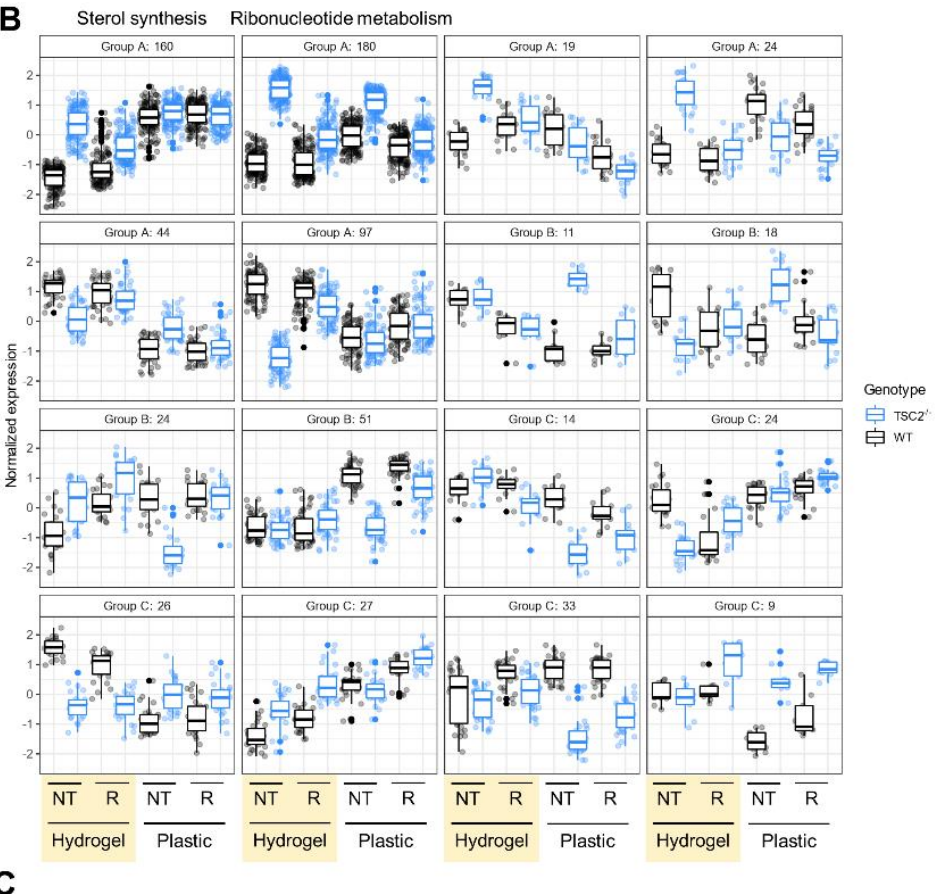

C

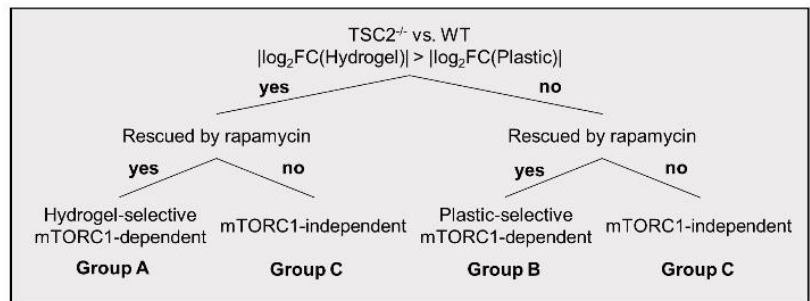

1402 Fig. S2. Hydrogel culture potentiates differential mTORC1-signalling between WT and

1403 TSC2 ${ }^{-/-}$cells. (A) Representative maximum intensity projection images used for quantification,

1404 following culture on hydrogel or plastic for three days $\pm 20 \mathrm{nM}$ rapamycin. Scale bars of $250 \mu \mathrm{m}$.

1405 (B) Gene clusters following hierarchal clustering of DEGs found significant (FDR < 0.05) in the

1406 interaction between genotype and ECM (761 genes). Clustering was based on the pattern of gene

1407 expression across the 8 employed conditions. The first two clusters are annotated to be enriched

1408 in sterol synthesis and ribonucleotide metabolism terms. (C) Classification scheme applied to the

1409 gene clusters visualized in (B). Labelling of Groups A, B, and C is for ease of visualization in

1410 (B) and does not confer any specific biological meaning. 
A

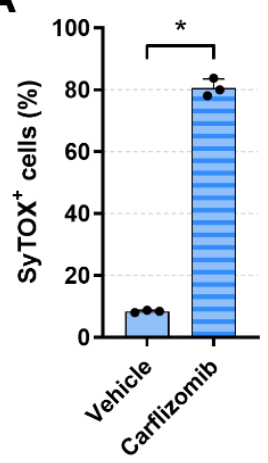

C

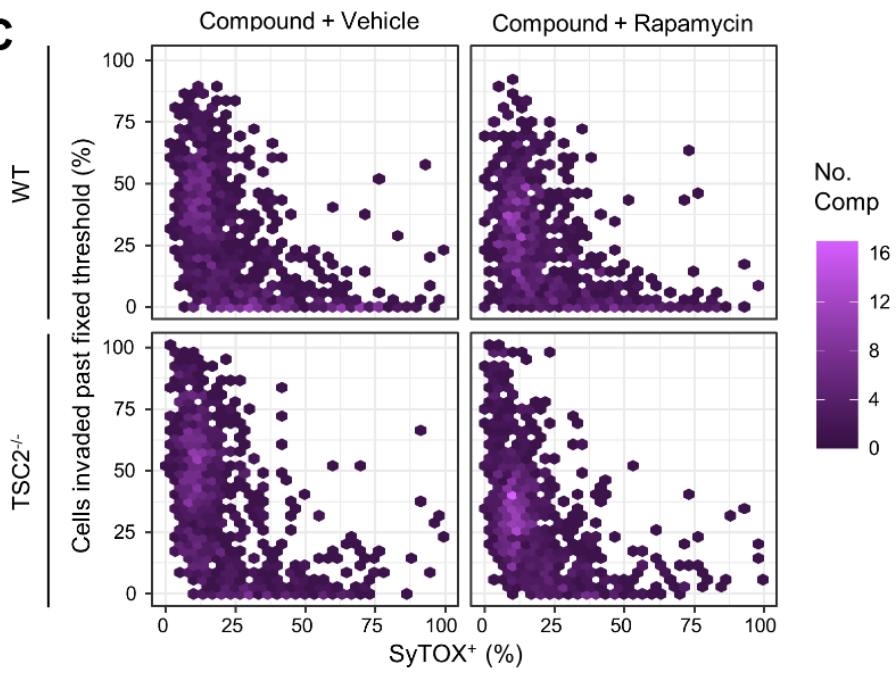

B

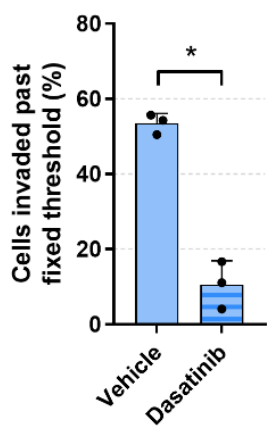

Comp

Fig. S3. Three-dimensional drug screen identifies HDAC inhibitors as anti-invasive and

1413 selectively cytotoxic towards TSC2 ${ }^{-/-}$LAM cells. (A) Percentage of SyTOX ${ }^{+}$TSC2 $^{-/-}$cells in

1414 hydrogel culture for three days $\pm 200 \mathrm{nM}$ carfilzomib (mean $\pm \mathrm{SD} ; *=\mathrm{p}<0.05$ by Student t-test;

$1415 \mathrm{n}=3$ ). (B) Percentage of $T S C 2^{-/}$cells invaded past fixed threshold (determined by median

1416 invasion distance of untreated controls), following three-day hydrogel culture $\pm 40 \mathrm{nM}$ dasatinib

1417 (mean $\pm \mathrm{SD} ;{ }^{*}=\mathrm{p}<0.05$ by Student $\mathrm{t}$-test; $\mathrm{n}=3$ ). (C) Compound invasion modulation plotted

1418 against cytotoxicity, separated by genotype and rapamycin treatment. Fixed threshold determined

1419 by median invasion distance of genotype-specific untreated controls. Hexagonal plot employed

1420 to demonstrate compound densities. (D) Waterfall plots of compound selective invasion $\mathrm{z}$-scores

1421 in ranked order; positive values indicate greater anti-invasive effects towards $T S C 2^{-/}$, negative 
1422 values indicate greater anti-invasive effects towards WT. Compounds conferring statistically

1423 significant selective invasion modulation highlighted in black. (E-F) Top 10 most statistically

1424 significant targets enriched in screen data, stratified by screen parameter. Enrichment analysis

1425 was performed via adaptation of the GSEA algorithm, using annotated targets of the compound

1426 library. 
A

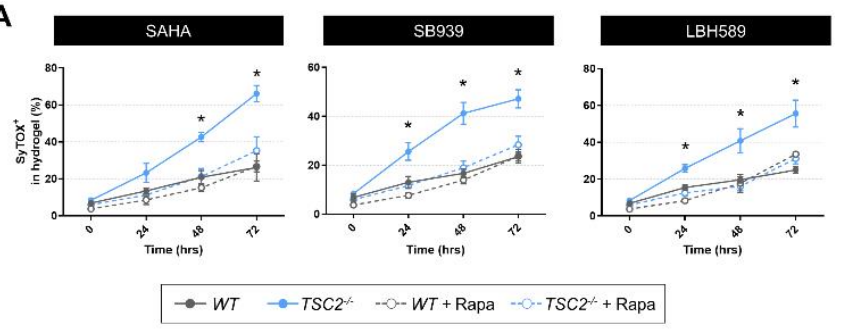

C
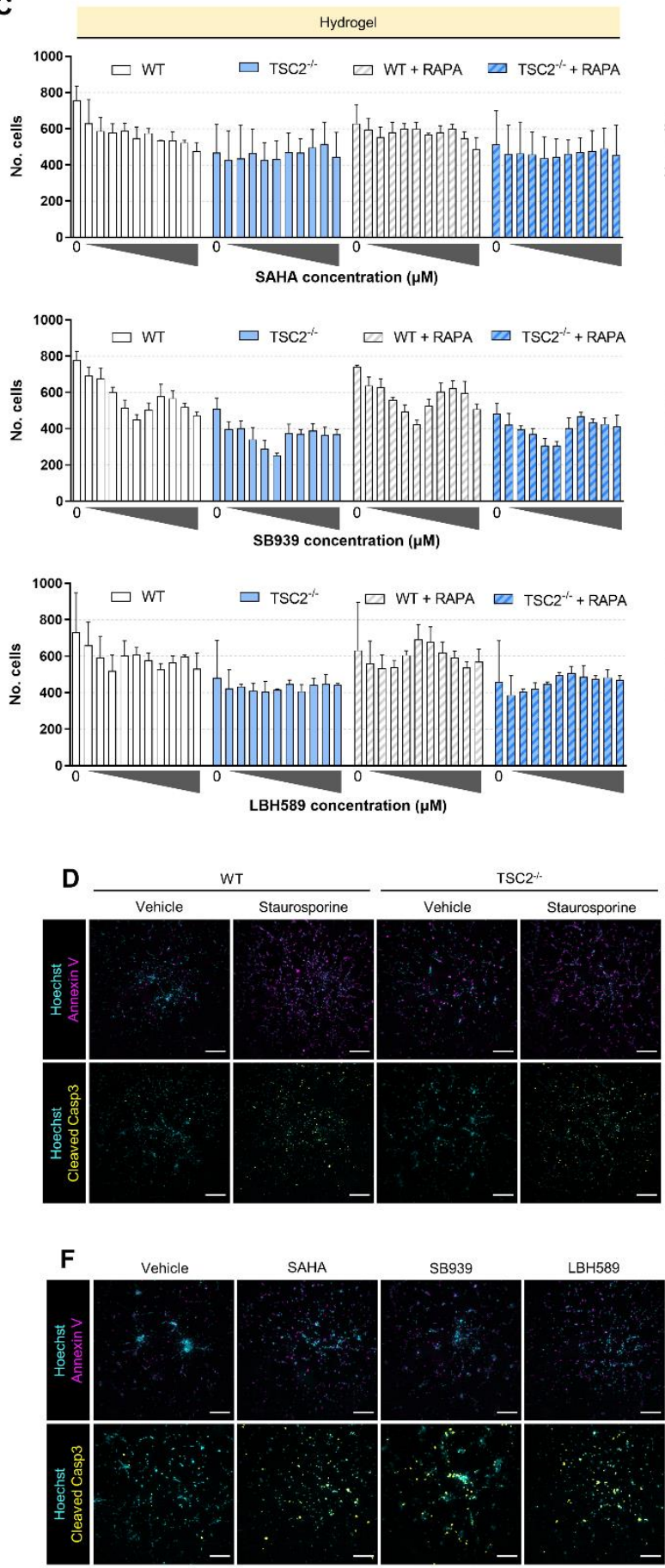

B
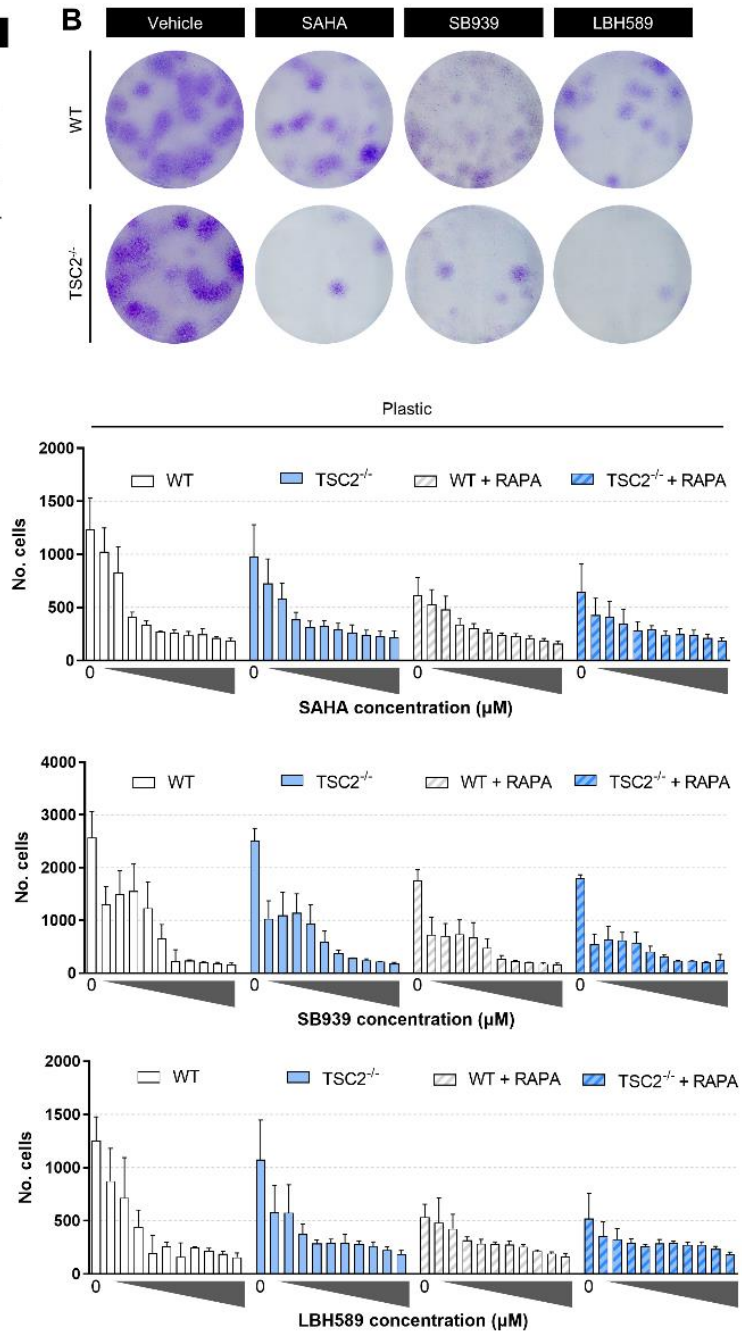

E
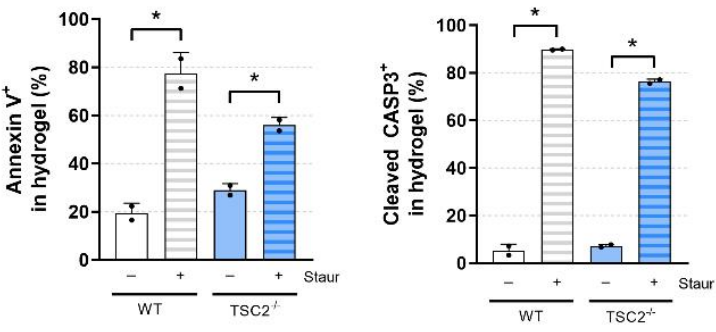

G

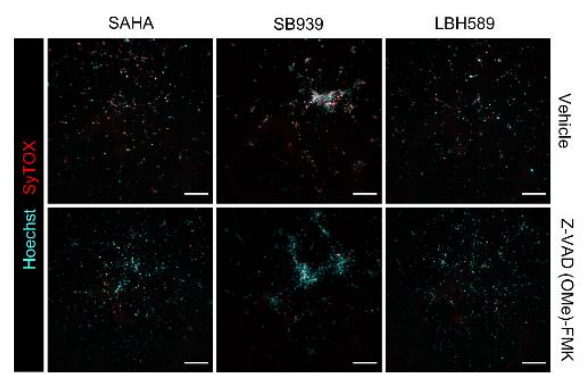


1428 Fig. S4. Three safe-in-human HDAC inhibitors induce mTORC1-dependent selective

1429 cytotoxicity exclusively in hydrogel culture. (A) Percentage of SyTOX ${ }^{+}$cells following time-

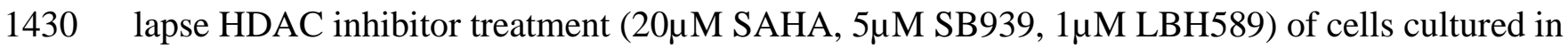

1431 hydrogel $\pm 20 \mathrm{nM}$ rapamycin (mean $\pm \mathrm{SD} ; *=\mathrm{p}<0.05$ by two-factor ANOVA with Tukey’s

1432 post-hoc comparison; $\mathrm{n}=3$ ). (B) Clonogenic assay following three-day HDAC inhibitor

1433 treatment $(20 \mu \mathrm{M}$ SAHA, $5 \mu \mathrm{M}$ SB939, $1 \mu \mathrm{M}$ LBH589) of cells cultured in hydrogel. After

1434 treatment, cells were extracted from hydrogel and replated in 2D to assess clonogenicity. (C)

1435 Number of cells detected in culture by high content imaging following three-day HDAC

1436 inhibitor treatment in hydrogel or plastic culture $\pm 20 \mathrm{nM}$ rapamycin. Inhibitor concentrations

1437 escalated in two-fold increments: SAHA $(0.31 \mu \mathrm{M} \min , 160 \mu \mathrm{M} \max ), \mathrm{SB} 939(0.04 \mu \mathrm{M} \min$,

$143820 \mu \mathrm{M} \max )$, and LBH589 (0.02 $\mu \mathrm{M}$ min, 10 $\mu \mathrm{M} \max )$. Mean $\pm \mathrm{SD}, \mathrm{n}=3$. (D-E) Representative

1439 maximum intensity projection images and quantification of live cell imaging dyes used in

1440 hydrogel culture, following $4 \mathrm{hr}$ treatment of $1 \mu \mathrm{M}$ staurosporine (mean $\pm \mathrm{SD} ; *=\mathrm{p}<0.05$ by

1441 Student t-test; $\mathrm{n}=2$ ). Scale bars of $250 \mu \mathrm{m}$. (F-G) Representative maximum intensity projection

1442 images of live cell imaging dyes used in hydrogel culture, following three-day treatment with

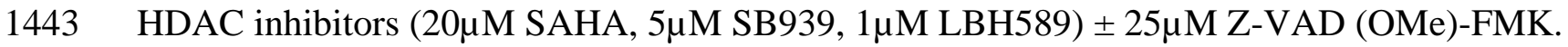

1444 Scale bars of $250 \mu \mathrm{m}$. 
A
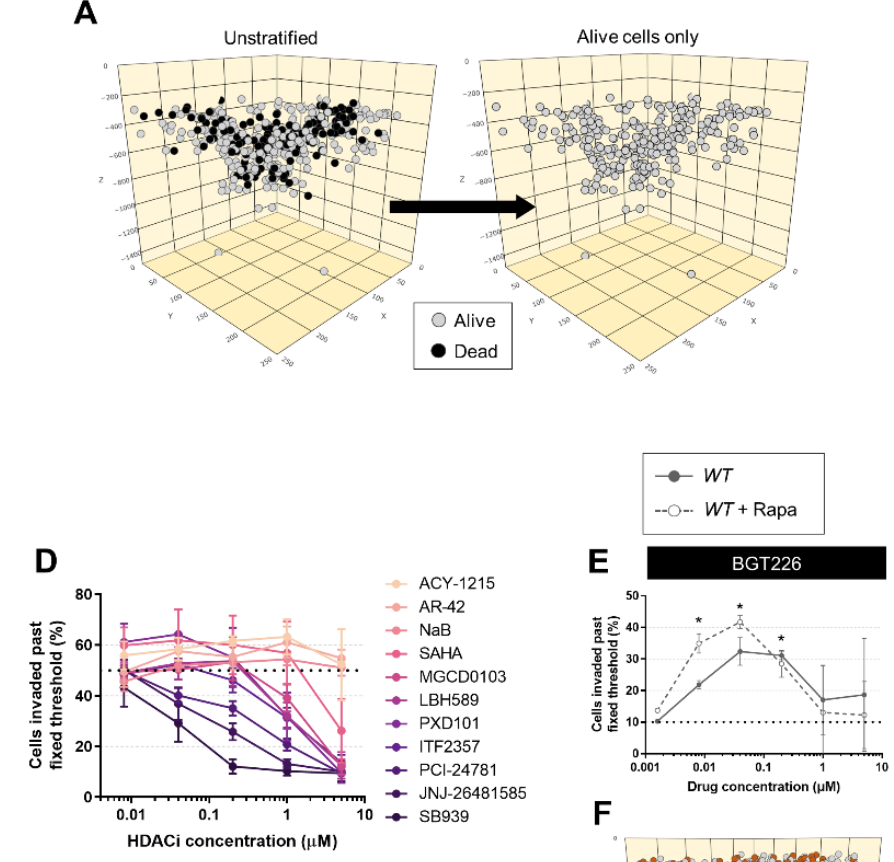

$\mathbf{F}$

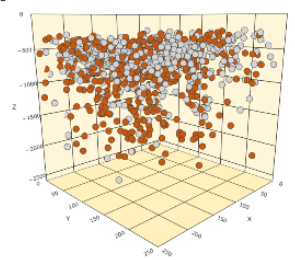

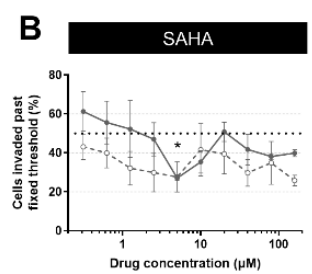
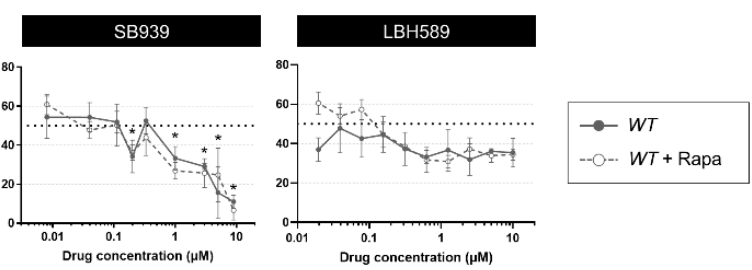

C
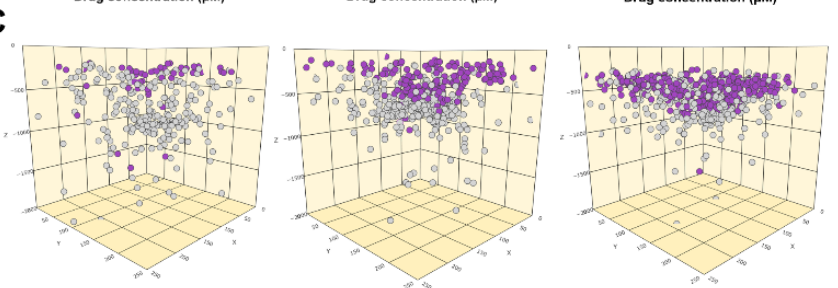

Vehicle

HDACi
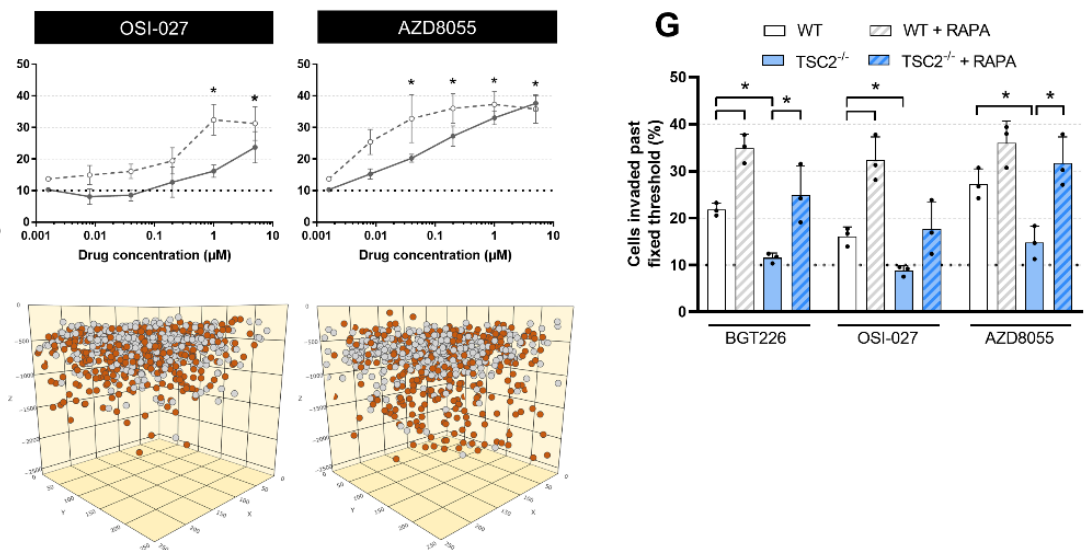

1445 Fig. S5. HDAC inhibitors attenuate cell invasion independent of cytotoxicity while mTOR

1446 inhibitors potentiate the invasion phenotype. (A) Schematic for removal of SyTOX $\mathrm{Sells}^{+}$to

1447 determine invasion distribution of live cells. (B) Live WT cells invaded past fixed threshold set

1448 by median invasion distance of vehicle control, upon three-day HDAC inhibitor treatment \pm

$144920 \mathrm{nM}$ rapamycin (mean $\pm \mathrm{SD} ; *=\mathrm{p}<0.05$ by ANOVA with Dunnett post-hoc comparison to

1450 untreated; $n=3$ ). (C) Computational reconstruction of live cell spatial positions upon three-day

1451 hydrogel culture of WT \pm HDAC inhibitor treatment (5 $\mu$ M SAHA, $5 \mu$ M SB939, $1 \mu$ M LBH589).

1452 Note that treated and untreated were in separate wells; cells were plotted in the same volume for

1453 ease of visualizing relative distances travelled. (D) Effect of 11 HDAC inhibitors on $T S C 2^{\%-}$ live

1454 cell invasion $\pm 20 \mathrm{nM}$ rapamycin. Fixed threshold set by median invasion distance of vehicle

1455 control. (E) Live WT cells invaded past fixed threshold set by $90^{\text {th }}$ percentile invasion distance of 
1456 vehicle control, upon three-day mTOR inhibitor treatment $\pm 20 \mathrm{nM}$ rapamycin $(\mathrm{mean} \pm \mathrm{SD} ; *=$

$1457 \mathrm{p}<0.05$ by ANOVA with Dunnett post-hoc comparison to untreated; $\mathrm{n}=3$ ). (F) Computational

1458 reconstruction of live cell spatial positions upon three-day hydrogel culture of WT \pm mTOR

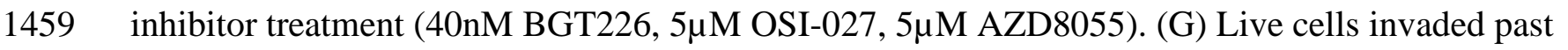

1460 fixed threshold set by $90^{\text {th }}$ percentile invasion distance of genotype-matched vehicle control,

1461 upon three-day mTOR inhibitor treatment (8nM BGT226, 1 $\mu$ M OSI-027, 200nM AZD8055) \pm

$146220 \mathrm{nM}$ rapamycin (mean $\pm \mathrm{SD} ; *=\mathrm{p}<0.05$ by Student t-test; $\mathrm{n}=3$ ). 
A

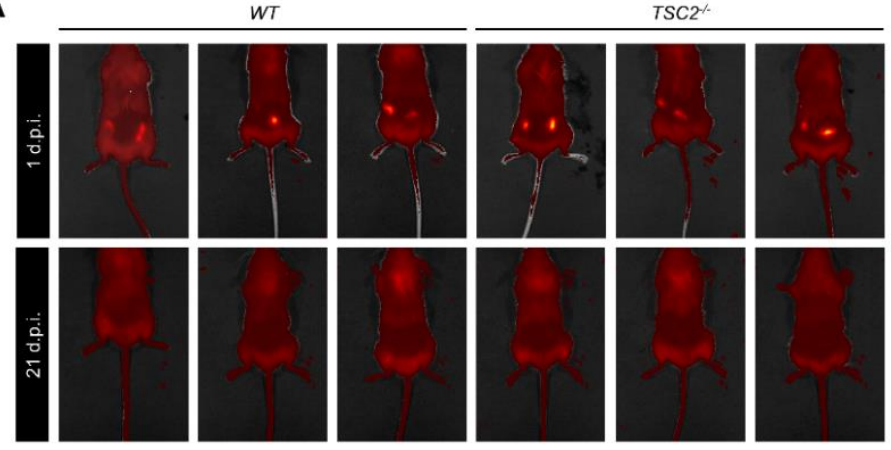

C

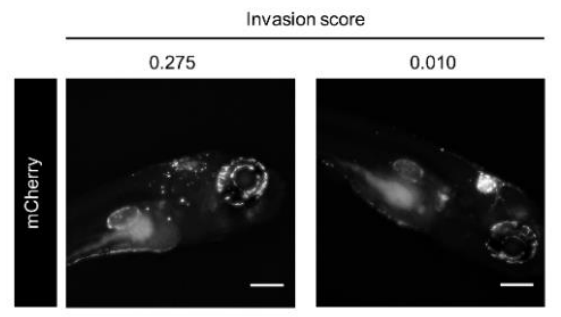

D

B
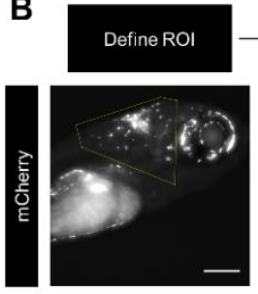

Threshold positive

signal

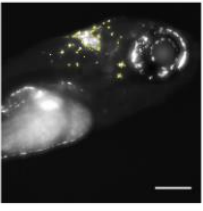

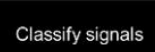

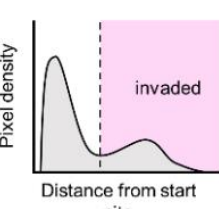

Sum intensities

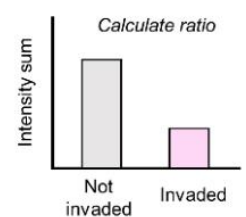

E

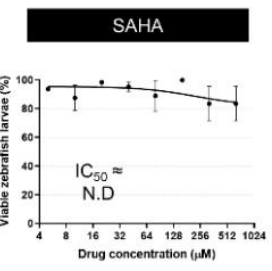

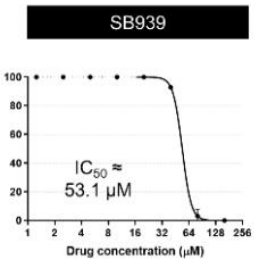
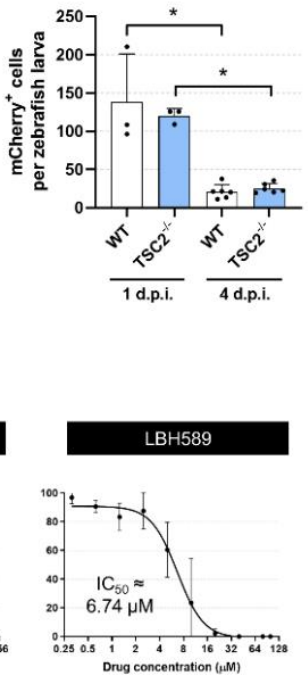

1463 Fig. S6. HDAC inhibitors are anti-invasive and selectively cytotoxic towards TSC2 $^{-/-}$cells

1464 xenotransplanted into zebrafish. (A) Visualization of mCherry $^{+}$cells by IVIS following

1465 subcutaneous transplantation into rear flanks of immunodeficient NSG mice. (B) Schematic

1466 representation of invasion score calculation in zebrafish larvae. See Supplementary Materials and

1467 Methods for more details. Scale bars of $200 \mu \mathrm{m}$. (C) Representative images of TSC2 $^{-/} \mathrm{mCherry}^{+}$

1468 cells disseminated 4 dpi with the associated invasion score. Scale bars of $200 \mu \mathrm{m}$. (D) Number of

1469 mCherry $^{+}$cells per zebrafish detected by flow cytometry following whole larvae dissociation at 1

1470 and 4 dpi. Each replicate is a pool of $15-20$ zebrafish larvae (mean $\pm \mathrm{SD} ; *=\mathrm{p}<0.05$ by

1471 Student t-test; $n=3-6$ ). (E) Dose-toxicity curves of zebrafish larvae treated with HDAC

1472 inhibitors by immersion therapy. 


\section{SUPPLEMENTARY TABLES}

1475 Table S1. Differential gene expression analysis of bulk RNA-seq data, untreated samples only.

1476 (A) DEG analysis of genotype (TSC2 ${ }^{-/-}$vs. WT), controlling for substrate covariate. (B) DEG

1477 analysis of substrate (hydrogel vs. plastic), controlling for genotype covariate. (C) DEG analysis

1478 of interaction between genotype and ECM.

1480 Table S2. GO term enrichment analysis of DEG lists. (A) GO term enrichment in TSC2 $^{-/}$vs. WT

1481 DEG list $\left(\right.$ FDR $\left.<0.05,\left|\log _{2} \mathrm{FC}\right|>1\right)$. (B) GO term enrichment in hydrogel vs. plastic DEG list

$1482\left(\mathrm{FDR}<0.05,\left|\log _{2} \mathrm{FC}\right|>1\right)$. (C) GO enrichment of genotype:substrate interaction DEG list (FDR

$1483<0.05)$.

1485 Table S3. Three-dimensional drug screen raw data. (A) Compound information from Ontario

1486 Institute of Cancer Research kinase inhibitor and tool compound libraries. (B-C) Cytotoxicity

1487 and invasion modulation effects of compounds, (B) statistic descriptions and (C) raw data.

1489 Table S4. Enrichment results via adaptation of GSEA. Results for statistics of (A) selective

1490 cytotoxicity, positive enrichment (i.e., selectively cytotoxic towards $T S C 2^{-/}$), (B) selective

1491 cytotoxicity, negative enrichment (i.e., selectively cytotoxic towards WT), (C) invasion

1492 modulation, positive enrichment (i.e., attenuate invasion), (D) invasion modulation, negative

1493 enrichment (i.e., potentiate invasion). 
bioRxiv preprint doi: https://doi.org/10.1101/2021.07.03.451004; this version posted July 4, 2021. The copyright holder for this preprint (which was not certified by peer review) is the author/funder, who has granted bioRxiv a license to display the preprint in perpetuity. It is made available under aCC-BY-NC 4.0 International license.

1495 Table S5. Elion ${ }^{\mathrm{TM}}$ structure-based compound analysis. (A-B) Significantly enriched targets and

1496 mechanisms of action by (A) selective cytotoxicity towards $T S C 2^{-/}$cells and (B) invasion

1497 attenuation. (C-D) Significantly enriched GO and PFAM terms (based on significantly enriched

1498 targets) by (C) selective cytotoxicity towards $T S C 2^{-/-}$cells and (D) invasion attenuation. 
bioRxiv preprint doi: https://doi.org/10.1101/2021.07.03.451004; this version posted July 4, 2021. The copyright holder for this preprint (which was not certified by peer review) is the author/funder, who has granted bioRxiv a license to display the preprint in perpetuity. It is made available under aCC-BY-NC 4.0 International license.

\section{SUPPLEMENTARY MOVIES}

1500

1501 Movie S1. Brightfield Z-stack of WT invading through the hydrogel, counterstained with

1502 Hoechst.

1503

1504 Movie S2. Brightfield Z-stack of $T S C 2^{-/-}$invading through the hydrogel, counterstained with 1505 Hoechst.

1506 